\title{
Laser-cooling limits and single-ion spectroscopy
}

\author{
D. J. Wineland, Wayne M. Itano, J. C. Bergquist, and Randall G. Hulet \\ Time and Frequency Division, National Bureau of Standards, Boulder, Colorado 80803
}

(Received 9 January 1987)

\begin{abstract}
The limitations to the achievement of low kinetic energies for laser cooling of single ions confined in electromagnetic traps are discussed. Sideband cooling of an ion in an $\mathrm{rf}$ (Paul) trap is reexamined including the effects of finite laser bandwidth and the energy of the rf micromotion. The micromotion is the oscillatory motion of the ion at the same frequency as the rf voltage applied to the trap electrodes. Sideband cooling of ions in a Penning trap is examined for the first time. In both cases, cooling to the zero-point energy of the ion in the trap should be possible and a method for verifying this condition is suggested. The implications for high-resolution, high-accuracy spectroscopy are investigated. Under certain conditions, the uncertainty in the second-order Doppler shift may be significantly less than 1 part in $10^{18}$.
\end{abstract}

\section{INTRODUCTION}

Over the past few years, the number of experimental and theoretical investigations of laser cooling has increased dramatically. $^{1-4}$ Much of this initial work has concentrated on characterizing the cooling process and understanding the lower limits to cooling. This is partly because the attainment of very low velocities will be important in applications such as high-resolution spectroscopy (reduction of Doppler shifts) and low-energy studies of atom-atom and atom-surface interactions. Minimum kinetic energies have often been expressed in terms of temperature. For free or weakly bound atomic particles, we make the identification $m\left\langle v_{i}^{2}\right\rangle / 2=k_{B} T_{i} / 2$ where $m\left\langle v_{i}^{2}\right\rangle / 2$ is the kinetic energy in the $i$ th degree of freedom, $T_{i}$ is the temperature for that degree of freedom, and $k_{B}$ is Boltzmann's constant. For atomic particles bound in a harmonic well, $T_{x}$ defined by the above relationship is therefore a measure of the average kinetic energy in the $x$ direction.

When the above relationship between kinetic energy and temperature holds, and when laser cooling is accomplished by driving a single photon transition, the minimum achievable temperature is given by the "Doppler" cooling limit (or "heavy particle" limit ${ }^{4}$ ),

$$
T_{D}=\hbar \gamma / 2 k_{B}
$$

In Eq. (1), $\gamma^{-1}$ is the radiative lifetime of the laser-cooling transition, $2 \pi \hbar$ is Planck's constant, and we have assumed that the incident radiation is well below saturation intensity. For atoms bound in a harmonic well with characteristic "vibration" or oscillation frequency $\omega_{v}$, the Doppler cooling limit applies when $\gamma \gg \omega_{v}$. The free-particle case is for the limit $\omega_{v} \rightarrow 0$, and thus Eq. (1) still applies. $T_{D}$ is typically in the range of $1 \mathrm{mK}$ for strongly allowed electric dipole transitions. For the case of stored ions, temperatures less than about $10 \mathrm{mK}$ have been achieved. ${ }^{5-8}$ The uncertainties in the measurements have often been consistent with the temperature being at the theoretical limit given by Eq. (1). The lowest measured temperatures have recently been achieved by $\mathrm{Chu}$ and co-workers using neutral sodium atoms. ${ }^{9}$ They demonstrated a temperature $\left(T=240_{-60}^{+200} \mu \mathrm{K}\right)$ equal to the Doppler cooling limit using three intersecting and mutually perpendicular, standing-wave laser beams which were tuned to give maximum cooling.

For trapped ions or atoms, cooling in the "sideband" limit (when $\gamma<<\omega_{v}$ ) is achieved by tuning the incident radiation to one of the lower motional sidebands of the atomic absorption. For example, in Fig. 1, the laser is assumed to be tuned to $\omega_{0}-\omega_{v}$, where $\omega_{0}$ is the transition frequency for the atom at rest. In this case, the minimum kinetic energy achieved is often given in terms of the mean occupation number $\left\langle n_{v}\right\rangle$ of the harmonic oscillator state for the atom or ion in the well. The brackets \langle\rangle denote the average over time or the average over an en-

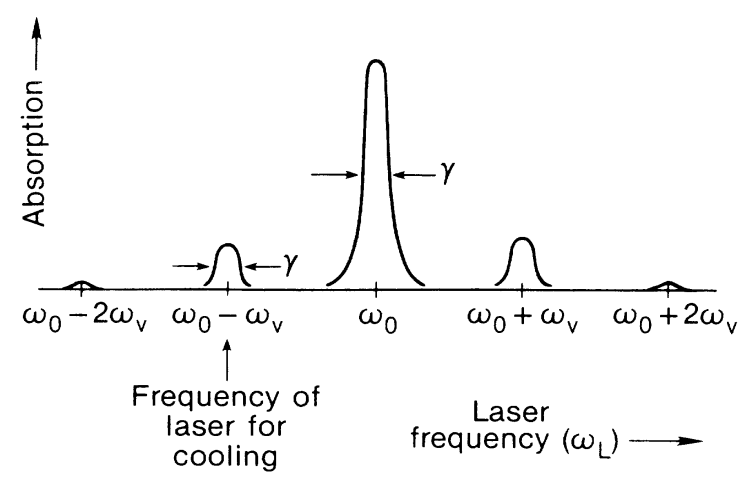

FIG. 1. Absorption spectrum of a single ion bound in a harmonic well, e.g., the absorption spectrum for the secular motion in an rf trap. It is assumed that $\omega_{v}$ (oscillation frequency of the ion in the harmonic well) $\gg \gamma$ (radiative linewidth of the cooling transition). The Lamb-Dicke limit is assumed, i.e., ion excursions are less than $\lambda / 2 \pi=c / \omega_{0}$. Therefore, the intensity of the sidebands is small compared to the carrier at $\omega_{0}$. Maximum cooling is achieved for a narrow-band laser (bandwidth less than $\gamma$ ) tuned to $\omega_{0}-\omega_{v}$. 
semble of identically prepared systems, assumed to be the same. In the sideband limit we have $e^{4,10,11}$

$$
\left\langle n_{v}\right\rangle_{s}=C_{s}\left(\gamma / \omega_{v}\right)^{2}
$$

In Eq. (2), $C_{s}$ is a constant on the order of 1 which depends on the atomic selection rules for the absorption and reemission. We have assumed that the incident radiation is well below saturation intensity. So far, sideband cooling has not been accomplished for trapped atoms or atomic ions. It has been realized in the special case of cooling the magnetron motion of trapped electrons, ${ }^{12}$ but in this case, the minimum achievable temperature is limited by thermal excitation. ${ }^{11,13}$

Since laser cooling gives rise to a thermal distribution of occupation numbers, ${ }^{4}$ we can write

$$
\left\langle n_{v}\right\rangle=\left[\exp \left(\hbar \omega_{v} / k_{B} T\right)-1\right]^{-1}
$$

Combining Eqs. (2) and (3), we achieve the minimum temperature in the sideband cooling limit,

$$
\begin{aligned}
T_{s} & \simeq \hbar \omega_{v} /\left[k_{B} \ln \left(\left\langle n_{v}\right\rangle^{-1}\right)\right] \\
& =\hbar \omega_{v} /\left[k_{B} \ln \left(\omega_{v}^{2} / C_{s} \gamma^{2}\right)\right] .
\end{aligned}
$$

When $\hbar \omega_{v}<<k_{B} T$, Eq. (3) reduces to the expression $m\left\langle v_{i}^{2}\right\rangle / 2=k_{B} T_{i} / 2$, which applies to the Doppler cooling limit of Eq. (1).

Equations (1) and (4) show that lower temperatures could be achieved by using weakly allowed transitions (smaller values of $\gamma$ ). In addition, lower temperatures could be achieved by using combinations of evaporative and adiabatic cooling and/or novel cooling schemes using more than one atomic transition or nonadiabatic manipulation of trap parameters. ${ }^{14-18}$ For example, several recent papers ${ }^{19-23}$ have considered cooling by using stimulated Raman transitions. Here the cooling limits of Eqs. (1) and (4) apply but $\gamma$ is now the linewidth of the stimulated Raman transition, which can be quite narrow. Other authors speculate about schemes to achieve temperatures as low as $10^{-10} \mathrm{~K}$ for free atoms. ${ }^{16}$

In this paper, we investigate the limits of laser cooling for single trapped ions. We also consider the implications of this cooling for accuracy in high-resolution spectroscopy. Some of the conclusions will also apply to the case of trapped neutral atoms but here we concentrate on the case of trapped ions. As we will try to demonstrate, describing the cooling limits in terms of temperature may not be particularly relevant for spectroscopic purposes. This may also be true for free neutral atoms. For example, if a single atom [mass of $100 \mathrm{u}$ (atomic mass units)] starts from rest, the time it takes it to reach a velocity $v$ corresponding to an effective temperature $T_{e}=10^{-10} \mathrm{~K}$ (via the relationship $\frac{1}{2} m v^{2}=\frac{1}{2} k_{B} T_{e}$ ) in the earth's gravitational field is about $10 \mu \mathrm{s}$. If we desired to maintain an effective temperature below $10^{-10} \mathrm{~K}$, the interrogation time between applications of cooling radiation would be quite short. For trapped ions or atoms, gravity need not play an important role; it merely shifts the origin of the trap. Therefore, barring other heating effects, interrogation times can be extremely long. Shifts in spectra caused by velocitychanging effects due to recoil can be made negligible. For example, the maximum of the recoil-free "carrier" (at frequency $\omega_{0}$ in Fig. 1) is shifted slightly due to the overlapping effects of the sidebands at $\omega_{0}-\omega_{v}$ and $\omega_{0}+\omega_{v}$ which are different in amplitude. ${ }^{11}$ (See Sec. V.) These pulling effects can be made extremely small.

Perhaps the most interesting case is when the ion or atom is cooled to near the zeroth quantum level, $\left\langle n_{v}\right\rangle<<1$. Independently of the method used, if $\left\langle n_{v}\right\rangle<<1$ can be achieved, the accuracies for highresolution spectroscopy need not be limited by motional effects. Since this condition can be achieved using laser cooling on single photon transitions, we investigate only this relatively simple case here. At the outset, unless specifically stated, we consider only a single ion in an $\mathrm{rf}$ or Penning trap. ${ }^{24,25}$ The reason is simply that for two or more ions in the trap, the limiting kinetic energies are considerably higher than those given by Eqs. (1)-(4). For example, for more than one ion in an $\mathrm{rf}$ trap, in order to overcome the Coulomb repulsion between ions, there must be a force on each ion from the trapping potential towards the center of the trap. This implies a certain kinetic energy of micromotion for each ion which can far exceed the kinetic energy of the secular motion. The micromotion $^{24,25}$ in an $\mathrm{rf}$ trap is the oscillatory motion of the ion(s) at the frequency of the imposed rf voltage applied to the electrodes. The secular motion is the additional, lower-frequency motion of the ion(s) due to the resulting pseudopotential well established by the spatially inhomogenous rf fields. It is this secular motion, at frequency $\omega_{v}$, which is cooled by laser cooling. The kinetic energy in the micromotion is directly related to the kinetic energy in the secular motion (Refs. 24 and 25 and Sec. II B) but is not directly cooled by the laser. For more than one ion in the trap, even though very low temperatures of the secular motion can be achieved through laser cooling, the kinetic energy would be dominated by the rf motion. ${ }^{24}$ For a single ion (Sec. II), this effect is considerably reduced. Similarly, for more than one ion in the Penning trap, the velocity in the rotation of the ion sample ${ }^{8,24}$ gives rise to a kinetic energy which can be greater than the limits of Eqs. (1)-(4). For a single ion this effect can be very small (Sec. III).

In Sec. II we reexamine the sideband cooling limit for ions in an $\mathrm{rf}$ trap where we include the effects of finite laser bandwidth and the importance of micromotion. In Sec. III, we examine sideband cooling in a Penning trap; this has not been done previously. In Sec. IV, we suggest a possible practical scheme for achieving $\left\langle n_{v}\right\rangle<<1$, and in Sec. V, we discuss a method for verifying that the zeroth quantum level is achieved. In Sec. VI we discuss the implications of these results for spectroscopy.

\section{SIDEBAND COOLING FOR A SINGLE ION IN AN rf (PAUL) TRAP}

\section{A. Laser cooling of secular motion in an rf trap}

We assume that the secular motion of the ion in the rf trap is harmonic and characterized by the frequencies $\omega_{x}$, $\omega_{y}$, and $\omega_{z}$ for the $x, y$, and $z$ directions, respectively. 
This case, or more generally the cooling of atoms trapped in a harmonic potential well, has been treated extensively in the literature. The most complete treatment appears to be that of Lindberg, Javanainen, and Stenholm, who include the effects of saturation.,26 However, lowest minimum temperatures are achieved with laser intensities below saturation, and simple perturbation methods ${ }^{10,11}$ can be used to find the minimum kinetic energies. Here, we use the formalism of Refs. 11 and 27, which treats each photon absorption and subsequent reemission as a scattering event-spontaneous Raman scattering. This process is spontaneous scattering because we assume low intensity and therefore the ion returns to its ground state via spontaneous emission. It is Raman scattering because when the internal atomic states and vibration states (oscillation states in the trap) are treated together, the ion can change the vibration quantum number $n_{v}$ in a scattering event. Let $n_{l x}$ denote the $n$th vibration state of the ion in the $x$ direction. The subscript $l$ means that this is the initial $(l$, lower) vibration state of the ion before scattering. Let $P\left(n_{l x}\right)$ be the probability that the ion is in the initial state with quantum number $n_{l x}$, where $\sum_{n_{l x}} P\left(n_{l x}\right)=1$. Assume that the ion is irradiated with a narrow-band laser (bandwidth $\Delta \omega_{L}<<\gamma$ ) directed along the $x$ direction. The average rate of energy change in the $i$ th direction due to this laser in the $x$ direction is given by

$$
\left\langle\dot{E}_{i x}\right\rangle=\dot{N}_{x} \sum_{n_{l x}, n_{j x}} P\left(n_{l x}\right)\left\{R f_{s i}+\delta_{i x}\left[E\left(n_{j x}\right)-E\left(n_{l x}\right)\right]\right\} \frac{\left|\left\langle n_{j x}\left|e^{i k_{x} x}\right| n_{l x}\right\rangle\right|^{2}}{1+\left[\frac{2}{\gamma}\left\{\omega_{0}-\omega_{L x}+\left[E\left(n_{j x}\right)-E\left(n_{l x}\right)\right] / \hbar\right\}\right]^{2}}
$$

This is a straightforward generalization of Eq. (48) of Ref. 11. $\dot{N}_{x}=I_{x} \sigma_{0 x} / \hbar \omega_{0}$ is the scattering rate for the laser tuned to resonance with the ion at rest, $\omega_{0}$ is the rest frequency of the ion, $R=(\hbar k)^{2} / 2 m$ is the recoil energy where $k$ is the photon wave vector $\left(k=k_{x} \simeq k_{y} \simeq k_{z}\right), m$ is the ion mass, $\hbar k \sqrt{f_{s i}}$ is the rms value of reemitted photon momentum along the $i$ th direction, ${ }^{27} E\left(n_{l x}\right)$ $=\hbar \omega_{x}\left(n_{l x}+\frac{1}{2}\right)$ where $\omega_{x}$ is the harmonic well frequency in the $x$ direction, $\delta_{i x}$ is the Kronecker $\delta$, and $\omega_{L x}$ is the laser frequency. The sum is over all possible initial vibrational states (with quantum numbers $n_{l x}$ ) and intermediate vibrational states $j$. The intermediate states are the states with the ion in the excited electronic state and with vibrational quantum number $n_{j x}$. Similar expressions hold for laser beams directed along the $y$ and $z$ directions.

Equation (5) holds for arbitrary ion kinetic energy (neglecting relativistic corrections). The most interesting case, however, is that for maximum laser cooling; that is, when the laser is tuned to the first lower sideband frequency $\omega_{0}-\omega_{x}$. For the final stages of cooling, we anticipate that the ion will be in the Lamb-Dicke regime. ${ }^{28}$ This condition is given by the relationship $\left(k^{2}\left\langle x^{2}\right\rangle\right)^{1 / 2}$ $=k x_{0}\left\langle 2 n_{x}+1\right\rangle^{1 / 2}<<1 \quad$ where $\left\langle n_{x}\right\rangle=\sum_{n_{l x}} P\left(n_{l x}\right) n_{l x}$. Qualitatively, the Lamb-Dicke regime implies that the amplitude of the ion's motion is less than $\lambda / 2 \pi$. $x_{0}=\left(\hbar / 2 m \omega_{x}\right)^{1 / 2}$ is the zero-point amplitude so that $k x_{0}=\left(R / \hbar \omega_{x}\right)^{1 / 2} \equiv \beta_{x}^{1 / 2}$. Hence, for the Lamb-Dicke limit to apply we must have $R \ll \hbar \omega_{x}$, or equivalently, $\omega_{x} \gg \omega_{R}$ where $\omega_{R}=R / \hbar$ is the recoil frequency. For $\lambda=300 \mathrm{~nm}$ and $m=100 \mathrm{u}, \omega_{R} / 2 \pi \simeq 22 \mathrm{kHz}$. For these conditions and for $\omega_{x} / 2 \pi=1 \mathrm{MHz}$, then $x_{0} \simeq 0.007 \mu \mathrm{m}$ and $\beta_{x}=0.022$. If the Lamb-Dicke limit applies, then $e^{i k x} \simeq 1+i k x$, and only three excited states $j$ contribute to the sum in Eq. (5). Therefore (see Fig. 1),

$$
\begin{aligned}
\left\langle\dot{E}_{i x}\right\rangle \simeq \dot{N}_{x}[ & \left\langle n_{x}\right\rangle \beta_{x}\left(R f_{s i}-\delta_{i x} \hbar \omega_{x}\right) F_{x}+R f_{s i} \gamma^{2} /\left(4 \omega_{x}^{2}\right) \\
& \left.+\left(R f_{s i}+\delta_{i x} \hbar \omega_{x}\right)\left(\left\langle n_{x}\right\rangle+1\right) \beta_{x} \gamma^{2} /\left(16 \omega_{x}^{2}\right)\right]
\end{aligned}
$$

In Eq. (6), we have now included the factor

$$
F_{x}=\int_{0}^{\infty} I_{x}^{\prime}(\omega) \frac{d \omega}{1+\left[\frac{2}{\gamma}\left(\omega_{0}-\omega_{x}-\omega\right)\right]^{2}}
$$

which allows for the fact that the laser spectral width may be larger than $\gamma$. In practice, it may be desirable to make the laser bandwidth much greater than $\gamma$ to allow for anharmonic contributions to or instabilities in $\omega_{x}$. For simplicity, we assume that this width is still much less than $\omega_{x} . I_{x}^{\prime}(\omega)$ is a normalized intensity such that $\int I_{x}^{\prime}(\omega) d \omega=1$. Therefore, the laser spectral intensity is given by $I_{x} I_{x}^{\prime}(\omega)$ where $I_{x}$ is the total intensity. The average scatter (or absorption) rate $\left\langle\dot{N}_{s}\right\rangle$ is related to Eq. (6) as

$$
\begin{aligned}
\left\langle\dot{N}_{s x}\right\rangle=\dot{N}_{x}[ & \left\langle n_{x}\right\rangle \beta_{x} F_{x}+\gamma^{2} /\left(4 \omega_{x}^{2}\right) \\
& \left.+\left(\left\langle n_{x}\right\rangle+1\right) \beta_{x} \gamma^{2} /\left(16 \omega_{x}^{2}\right)\right] .
\end{aligned}
$$

If we also have laser beams directed along the $y$ and $z$ axes, equations analogous to Eqs. (5)-(8) hold for $\left\langle\dot{E}_{i y}\right\rangle$, $\left\langle\dot{N}_{s y}\right\rangle,\left\langle\dot{E}_{i z}\right\rangle$, and $\left\langle\dot{N}_{s z}\right\rangle$.

Case 1. Suppose we have only one laser beam (say, along the $x$ axis) but that the $x, y$, and $z$ degrees of freedom are thermalized to the same temperature. This would correspond to the case of more than one ion in the trap where Coulomb coupling provides the thermalization, but where we must assume that the individual ion oscillation frequencies are not significantly perturbed. That is, we neglect the effects of space-charge repulsion. The total rate of energy change is given by Eq. (6) as

$$
\begin{aligned}
\langle\dot{E}\rangle & =\sum_{i(=x, y, z)}\left\langle\dot{E}_{i x}\right\rangle \\
& \simeq \dot{N}_{x}\left\{\left\langle n_{x}\right\rangle\left[-R F_{x}+R \gamma^{2} /\left(16 \omega_{x}^{2}\right)\right]+5 R \gamma^{2} /\left(16 \omega_{x}^{2}\right)\right\},
\end{aligned}
$$

where terms of higher order in $\beta_{x}$ have been neglected. 
In steady state $(\langle\dot{E}\rangle=0)$, we have

$$
\left\langle n_{x}\right\rangle=5 \gamma^{2}\left\{16 \omega_{x}^{2}\left[F_{x}-\gamma^{2} /\left(16 \omega_{x}^{2}\right)\right]\right\}^{-1} .
$$

We will assume throughout that the spectral density of the laser is fairly uniform with width $\Delta \omega_{L}$. If the spectral width of the laser is larger than $\gamma$, we can get an estimate of $F$ by assuming that the laser spectrum is flat with width $\Delta \omega_{L}$, that is, $I_{x}^{\prime}=1 / \Delta \omega_{L}$. For $\Delta \omega_{L} \gg \gamma$, Eq. (7) gives $F_{x}=\pi \gamma / 2 \Delta \omega_{L}$. Since we have assumed $\Delta \omega_{L} \ll<\omega_{x}$, then $F \gg \gamma^{2} / \omega_{x}^{2}$ and we can neglect the $\gamma^{2} /\left(16 \omega_{x}^{2}\right)$ term in the previous expression and obtain

$$
\left\langle n_{x}\right\rangle=5 \gamma^{2} /\left(16 \omega_{x}^{2} F_{x}\right) .
$$

If the laser linewidth is much less than $\gamma, F_{x}=1$ yielding $\left\langle n_{x}\right\rangle=5 \gamma^{2} / 16 \omega_{x}^{2}$, which is the result of Ref. 11 .

Equation (10) is valid in the Lamb-Dicke limit, $R / \hbar \omega_{x}<1$. To find corrections to this expression which are first order in $\beta_{x}=R / \hbar \omega_{x}$ we must include matrix elements $\left\langle n_{x}-2\left|e^{i k x}\right| n_{x}\right\rangle$ and $\left\langle n_{x}+2\left|e^{i k x}\right| n_{x}\right\rangle$ in Eq. (5) and take $e^{i k x}=1+i k x-k^{2} x^{2} / 2$. In this case we obtain

$$
\left\langle n_{x}\right\rangle=\frac{5}{16} \frac{\gamma^{2}}{\omega_{x}^{2}}\left(1+\frac{22}{45} \beta_{x}\right) / F_{x} .
$$

Case 2. Suppose we have three laser beams directed along the $x, y$, and $z$ axes which are tuned to the appropriate first lower sidebands for each direction. We must write an equation for $\left\langle\dot{E}_{x}\right\rangle$, the rate of energy change in the $x$ direction, using Eq. (6), and the analogous equations which give contributions to $\left\langle\dot{E}_{x}\right\rangle$ from the $y$ and $z$ beams. Similarly, we must write equations for $\left\langle\dot{E}_{y}\right\rangle$ and $\left\langle\dot{E}_{z}\right\rangle$. In steady state we can solve these three equations for $\left\langle n_{x}\right\rangle$, $\left\langle n_{y}\right\rangle$, and $\left\langle n_{z}\right\rangle$. These expressions are somewhat complicated but reflect the basic idea that if one of the scatter rates is large, say $\dot{N}_{x} \gg \dot{N}_{y}, \dot{N}_{z}$, and if $f_{s x} \simeq f_{s y} \simeq f_{s z}$ and $\omega_{x} \simeq \omega_{y} \simeq \omega_{z}$, then $\left.\left\langle n_{y}\right\rangle,\left\langle n_{z}\right\rangle\right\rangle\left\langle\left\langle n_{x}\right\rangle\right.$ due to the recoil heating in the $y$ and $z$ directions from the laser directed along the $x$ direction. If, however, we examine the special case where $\dot{N}_{i} \simeq \dot{N}_{j}, \omega_{i} \simeq \omega_{j}, F_{i} \simeq F_{j}$, and $f_{s i} \simeq f_{s j} \simeq \frac{1}{3}$ $(i, j=x, y, z)$, then $\left\langle n_{x}\right\rangle \simeq\left\langle n_{y}\right\rangle \simeq\left\langle n_{z}\right\rangle$ where $\left\langle n_{x}\right\rangle$ is given by Eq. (10). In this case, laser cooling and recoil heating are approximately equal in the $x, y$, and $z$ directions.

Case 3. Suppose we have one laser beam (intensity $I$ ) at some oblique angle with respect to the $x, y$, and $z$ axes. If $f_{s x} \simeq f_{s y} \simeq f_{s z}$, then it is natural to choose $\mathbf{k}$ parallel to $\widehat{\mathbf{x}}+\hat{\mathbf{y}}+\widehat{\mathbf{z}}$ in order to avoid excessive recoil heating along any axis. This is also a natural choice for a typical ion trap where the inner ring diameter is equal to $\sqrt{2}$ times the endcap-to-endcap separation. Here, the $\hat{\mathbf{x}}+\hat{\mathbf{y}}+\hat{\mathbf{z}}$ direction splits the gap between ring and endcaps. Thus the $\widehat{\mathbf{x}}+\widehat{\mathbf{y}}+\widehat{\mathbf{z}}$ direction is a practical choice for introducing laser beams into the trap. In this case, $\dot{N}_{x}$ from Eqs. (5) and (6) is equal to $\dot{N} / 3$ where $\dot{N}=I \sigma_{0} / \hbar \omega_{0}$ is equal to the scatter rate for a narrow-band $\left(\Delta \omega_{L}<<\gamma\right)$ laser tuned to resonance $\left(\omega_{L}=\omega_{0}\right)$ for the ion at rest. Assume that $\omega_{x} \simeq \omega_{y} \simeq \omega_{z} \simeq \omega_{v}$ and that the laser bandwidth is broad enough to cover all three of the first lower sidebands, i.e., $F_{x} \simeq F_{y} \simeq F_{z}=F$. To achieve the best laser cooling, we must have $\left|\omega_{i}-\omega_{j}\right| \gtrsim R \dot{N} F / \hbar \omega_{v}(i \neq j)$. The reason for this can be seen by first assuming $\omega_{x}=\omega_{y}$. In this case the choice of the $x$ and $y$ axes is arbitrary. If we chose new axes where $\hat{\mathbf{y}}^{\prime}=(\hat{\mathbf{x}}-\hat{\mathbf{y}}) / \sqrt{2}$ so that $\hat{\mathbf{y}}^{\prime} \cdot \mathbf{k}=0$; this implies recoil heating without bound in the $\hat{\mathbf{y}}^{\prime}$ direction. ${ }^{11}$ For $\omega_{x} \neq \omega_{y}$, again assume $\mathbf{k}$ is parallel to $\widehat{\mathbf{x}}+\widehat{\mathbf{y}}+\widehat{\mathbf{z}}$, and choose $f_{s x} \simeq f_{s y} \simeq f_{s z}=f \simeq \frac{1}{3}$ (condition for isotropic scattering). Recoil heating occurs at a rate $R f \dot{N}_{s}$ in the $\hat{\mathbf{y}}^{\prime}$ direction (i.e., perpendicular to the laser beam) where $\dot{N}_{s}$ is the total scattering rate from the ion. For kinetic energies near the sideband cooling limit, the dominant scattering occurs in the wings of the carrier (at frequency $\omega_{0}$ ) of the ion absorption spectrum so that $\dot{N}_{s} \simeq \dot{N} \gamma^{2} / 4 \omega_{v}^{2}$ [see Eq. (8)]. The closer $\omega_{x}$ is to $\omega_{y}$, the higher is the energy in the $\hat{\mathbf{y}}^{\prime}$ direction. To estimate this energy, we consider the following. In the absence of laser scattering, if at some time $t=0$ all of the ion's energy (above the zeropoint energy) is in the $\hat{\mathbf{y}}^{\prime}$ direction, then after a time $\tau$ such that $\left|\omega_{x}-\omega_{y}\right| \tau=\pi$, all of the energy is in the $\hat{\mathbf{x}}^{\prime}=(\widehat{\mathbf{x}}+\widehat{\mathbf{y}}) / \sqrt{2}$ direction. This situation is like the case of two coupled oscillators (here, in the $\hat{\mathbf{x}}^{\prime}$ and $\hat{\mathbf{y}}^{\prime}$ directions) which exchange energy in time $\tau$. Therefore, the decay of energy from the $\hat{\mathbf{y}}^{\prime}$ direction [denoted $E\left(\hat{\mathbf{y}}^{\prime}\right)$ ] is approximately equal to $E\left(\hat{\mathbf{y}}^{\prime}\right) / \tau$. In steady state, we have $R f \dot{N}_{s} \simeq\left[E\left(\hat{\mathbf{y}}^{\prime}\right)-E\left(\hat{\mathbf{x}}^{\prime}\right)\right] / \tau$. If we desire to keep $\left[E\left(\hat{\mathbf{y}}^{\prime}\right)-E\left(\widehat{\mathbf{x}}^{\prime}\right)\right]<\left\langle n_{x}\right\rangle \hbar \omega_{v}$ due to the recoil heating in the $\hat{\mathbf{y}}^{\prime}$ direction, then we require $R f \dot{N}_{s} \tau<\left\langle n_{x}\right\rangle \hbar \omega_{v}$ or approximately

$$
\left|\omega_{x}-\omega_{y}\right| \gtrsim \dot{N} R F / \hbar \omega_{v} .
$$

From the above arguments, it is clear that we must also make the same restriction on $\left|\omega_{x}-\omega_{z}\right|$ and $\left|\omega_{y}-\omega_{z}\right|$. The condition in Eq. (12) can also be obtained by assuming that we want $\left|\omega_{i}-\omega_{j}\right|$ to be larger than the inverse of the time constant for laser cooling. ${ }^{5}$ In an axially symmetric rf trap, $\omega_{x}=\omega_{y}$. Therefore, in order to achieve the condition given by Eq. (12), the axial symmetry must intentionally be destroyed. ${ }^{5}$ Thus, if the condition of Eq. (12) holds, Eq. (6) and the analogous equations for $\left\langle\dot{E}_{i y}\right\rangle$ and $\left\langle\dot{E}_{i z}\right\rangle$ in steady state give

$$
\left\langle n_{i}\right\rangle=\left(\frac{12 f_{s i}+1}{16 F}\right)\left(\gamma / \omega_{v}\right)^{2} .
$$

Equation (13) reduces to Eq. (10) for $f_{s i}=\frac{1}{3}$. If the laser spectrum is uniform with bandwidth $\Delta \omega_{L}\left(I^{\prime}=1 / \Delta \omega_{L}\right)$, and $\Delta \omega_{L} \gg \gamma$,

$$
\left\langle n_{i}\right\rangle=\left(\frac{12 f_{s i}+1}{8 \pi}\right) \gamma \Delta \omega_{L} / \omega_{v}^{2} .
$$

The right-hand side of Eq. (14) is larger than Eq. (2) by a factor of approximately $\Delta \omega_{L} / \gamma$. As an example, assume $\omega_{v} / 2 \pi=1 \mathrm{MHz}, \Delta \omega_{L} / 2 \pi \simeq 100 \mathrm{kHz}, \gamma / 2 \pi=1 \mathrm{kHz}$, and $f_{s i}=\frac{1}{3}$. Then $\left\langle n_{i}\right\rangle \simeq 2 \times 10^{-5}$.

The meaning of $\left\langle n_{i}\right\rangle<<1$ is the following. After most photon scattering events, the ion's energy in the well is equal to $3 \hbar \omega_{v} / 2$, the zero-point energy. That is, most of the time the ion scatters a photon without recoil energy being imparted to the oscillation in the well. Infrequently, after some scattering events, the ion's energy in the well is equal to $(3 / 2+1) \hbar \omega_{v}$, that is, the ion recoils into the first harmonic oscillator state, for some direction. 
Once in the first harmonic oscillator state, it is pumped by the sideband cooling mechanism back to the zero-pointenergy state. That the ion does not assume some intermediate energy state in the well is reflected in the discrete spectrum of fluorescence. ${ }^{11}$ We also note that when the ion spontaneously emits a photon, its energy, when averaged over many scattering events, increases by $R$ per emission event, ${ }^{11}$ even though after any given scattering event, the vibrational energy can only change by zero or some multiple of $\hbar \omega_{v}$. Therefore, on the average, approximately $\hbar \omega_{v} / R$ scattering events occur before the ion recoils into the first harmonic oscillator state. With very high probability, the next scattering event returns the ion to the zero-point energy state.

Therefore, most of the time, the ion is in the zero-point energy state and photons are being scattered at a rate $\dot{N}_{s}=\dot{N} \gamma^{2} / 4 \omega_{v}^{2}$ (scattering in the wings of the carrier). In order to minimize the relative time the ion spends in the $n_{v}=1$ state and therefore minimize the ion's kinetic energy [i.e., make Eq. (14) valid], we must avoid saturation of the first lower sideband when the ion is in the $n_{v}=1$ state. From Eq. (8), we require $\dot{N} \beta F / 3<\gamma$. This implies that the scattering in the wings of the carrier $\dot{N}_{s}$ is less than $3 \gamma^{3} / 4 F \omega_{v} \omega_{R}$. For the conditions of our previous example $\left(\omega_{v} / 2 \pi=1 \mathrm{MHz}, \gamma / 2 \pi=1 \mathrm{kHz}, \omega_{R} / 2 \pi=22 \mathrm{kHz}\right)$, $\dot{N}_{s} \lesssim 0.2 / F$, which is small for $F$ near 1 .

\section{B. Limits of micromotion energy}

In Sec. II A, we have discussed the limits to laser cooling for an ion or atom in a purely harmonic well, e.g., for the secular motion of an ion in an rf trap. When an ion is confined in an rf trap, one must also consider the energy in the micromotion. ${ }^{24,25}$ If we treat the motion classically, one can show that the average kinetic energy in the micromotion is equal to the average kinetic energy in the secular motion for two trap configurations of practical interest: ${ }^{24,25}$ (1) when there is no static potential applied between the ring and end caps $\left(\omega_{x}=\omega_{y}=\omega_{z} / 2\right)$ or (2) when the appropriate static potential is applied between ring and end caps to give a nominally spherical trap $\left(\omega_{x}=\omega_{y} \simeq \omega_{z}\right)$. For other cases, this equality of micromotion and secular energies does not strictly hold, but the key point is that they are of the same order.

Since the very low kinetic energies predicted by Eqs. (2), (10), and (13) require a quantum-mechanical treatment of the ion motion, it is useful to address the question of micromotion energy again, but now treating the ion motion in an rf trap quantum mechanically. Here, we examine only the case of zero static potential applied to the trap electrodes. For the quantum treatment, Cook et al. ${ }^{29}$ have examined the solution to Schrödinger's equation for the $\mathrm{rf}$ trap in the form

$$
i \hbar \frac{\partial}{\partial t} \psi=-\frac{\hbar^{2}}{2 m} \nabla^{2} \psi+v(\mathbf{x}) \cos (\Omega t) \psi
$$

where $v(\mathbf{x}) \cos (\Omega t)$ is the potential inside the trap. By writing the wave function in the form

$$
\psi(\mathbf{x}, t)=g(\mathbf{x}, t) u(\mathbf{x}, t),
$$

where

$$
u(\mathbf{x}, t)=\exp [-i v(\mathbf{x}) \sin (\Omega t) / \hbar \Omega],
$$

Cook et al. ${ }^{29}$ show that we can identify $g$ with the secular motion. If we take the expectation value of the kinetic energy, $E_{K}=-\hbar^{2} \nabla^{2} / 2 m$, and average over one period of the drive frequency, we obtain

$$
\left\langle\psi\left|E_{K}\right| \psi\right\rangle_{\mathrm{av}}=\frac{1}{4 m \Omega^{2}} \int d^{3} x \nabla v \cdot \nabla v g^{*} g+\int d^{3} x E_{K} g^{*} g .
$$

The first term on the right-hand side of Eq. (18) is the expectation value of the effective potential (pseudopotential), calculated with the wave function $g$. The second term is the expectation value of the kinetic energy calculated with the wave function $g$. The net result is the same as in the classical case. That is, the average value of the effective potential energy $\left\langle(\nabla v)^{2}\right\rangle / 4 m \Omega^{2}$ (pseudopotential energy) is equal to the kinetic energy of the micromotion. In the simple case of a quadratic pseudopotential, this contribution is equal to the kinetic energy of the secular motion.

In an experimental situation, the kinetic energy in the micromotion is typically larger than indicated above. Sometimes there can be contact potential differences, or differences in stray charge buildup, between the electrodes. In the experiments using $\mathrm{Ba}^{+}, \mathrm{Mg}^{+}$, or $\mathrm{Be}^{+}$, contact potential differences on the electrodes might result from nonuniform deposition on the trap electrodes from the source of these atoms. ${ }^{30}$ For example, suppose one endcap has a surface which has a higher contact potential than the other two electrodes. This implies that in addition to the usual rf trapping forces, there is an additional steady force along the $z$ axis which shifts the center of the trap. The new center of the trap is given by the condition that the total average force on the ion is zero. Thus, the steady force due to the contact potential must be balanced by the pseudopotential force; hence, there is a resultant micromotion at the new center of the trap where the secular motion amplitude is zero (i.e., the position of the ion when it is cold). To estimate classically the size of this effect, we write the potential inside the trap due to applied voltages in the form ${ }^{24,25}$

$$
\phi(r, z)=\frac{U_{0}+V_{0} \cos (\Omega t)}{r_{0}^{2}+2 z_{0}^{2}}\left(r^{2}-2 z^{2}\right),
$$

where $U_{0}$ and $V_{0}$ are the static and time-varying voltages applied between the ring and end caps and where $2 r_{0}$ and $2 z_{0}$ are the internal ring diameter and endcap-to-endcap spacing as indicated in Fig. 2. (Effects of truncating the electrodes are neglected.) This gives rise to a pseudopotential of the form ${ }^{25}$

$$
\begin{aligned}
\phi_{p}(\bar{r}, \bar{z})= & \left(\frac{q V_{0}^{2}}{m \Omega^{2}\left(r_{0}^{2}+2 z_{0}^{2}\right)^{2}}+\frac{U_{0}}{r_{0}^{2}+2 z_{0}^{2}}\right) \bar{r}^{2} \\
& +\left(\frac{4 q V_{0}^{2}}{m \Omega^{2}\left(r_{0}^{2}+2 z_{0}^{2}\right)^{2}}-\frac{2 U_{0}}{r_{0}^{2}+2 z_{0}^{2}}\right) \bar{z}^{2} \\
& =\frac{m \omega_{r}^{2}}{2 q} \bar{r}^{2}+\frac{m \omega_{z}^{2}}{2 q} \bar{z}^{2},
\end{aligned}
$$




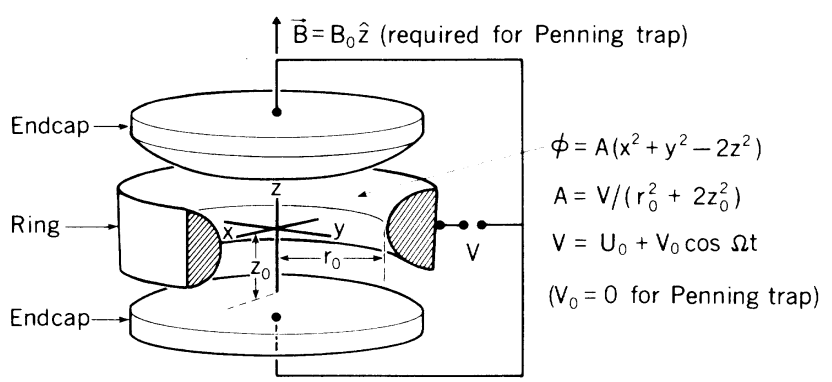

FIG. 2. Electrode configuration for an rf (Paul) or Penning trap. The inner surfaces of the electrodes are assumed to be equipotenitals of $\phi$, and the effect of truncating the electrodes is neglected. $r_{0}$ is the inner radius of the ring electrode and $2 z_{0}$ is the endcap-to-endcap spacing.

where $\omega_{r}=\omega_{x}=\omega_{y}$ and $\bar{r}$ and $\bar{z}$ are the radial and axial positions of the ion averaged over one cycle of the $\mathrm{rf}$ drive, that is, over a time $2 \pi / \Omega$. The $z$ force towards the center of the trap due to the pseudopotential is given by $F_{z}=-q \partial \phi_{p} / \partial \bar{z}$. The force away from the center of the trap due to a contact potential on one endcap is given by the following argument. Suppose we have a voltage $\Delta V$ on one endcap. This can be written as the sum of symmetric and antisymmetric terms as indicated in Fig. 3(a). The $z$ electric field due to the antisymmetric term can be approximated $^{31}$ by a parallel-plate capacitor of separation $2 z_{0} / \alpha$ as shown in Fig. 3(b). The factor $\alpha$ has been numerically estimated ${ }^{32,33}$ to be about 0.8 for a trap where $r_{0}^{2}=2 z_{0}^{2}$. If we assume the case of an $\mathrm{rf}$ trap with zero intentionally applied voltage $U_{0}$, then the center of the trap is given by the condition $F_{z}=-q \partial \phi_{p} / \partial \bar{z}-q \alpha \Delta V / 2 z_{0}$ $=0$, or

$$
\bar{z}=-\frac{q \alpha \Delta V}{2 m \omega_{z}^{2} z_{0}} .
$$

From this expression, we can find the kinetic energy in

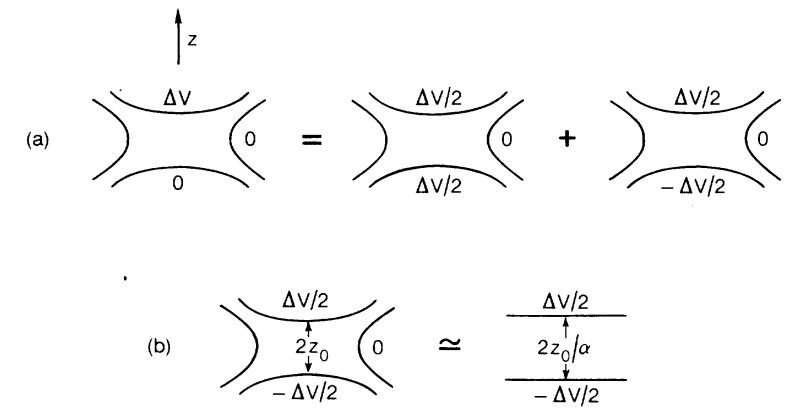

FIG. 3. Part (a) indicates how a potential $\Delta V$ applied to one endcap electrode can be viewed as the sum of voltages applied symmetrically and antisymmetrically to the electrodes. For ions near the center of the trap, the lowest-order contribution to the electric field from the antisymmetric part can be viewed as arising from a voltage $\Delta V$ applied between a parallel plate capacitor of separation $2 z_{0} / \alpha$. This is indicated in part (b) of the figure. From Refs. 32 and 33, $\alpha \simeq 0.8$. the micromotion $E_{K \mu}$ by recalling that it is equal to the pseudopotential energy, that is, $E_{K \mu}=q \phi_{p}$. Therefore, for the $z$ motion we have

$$
E_{K \mu}(z)=\left(\frac{q \alpha \Delta V}{\omega z_{0}}\right)^{2} / 8 m .
$$

To get an idea of the size of this effect, we examine the conditions of the miniature rf trap used to store single $\mathrm{Hg}^{+}$ions. $^{7}$ There, $\omega_{z} / 2 \pi \simeq 1.5 \mathrm{MHz}, z_{0} \simeq 320 \mu \mathrm{m}$, $r_{0} \simeq 450 \mu \mathrm{m}$. If $\Delta V=1 \mathrm{~V}, E_{K \mu}(z) \simeq 7 \times 10^{5} \hbar \omega_{z}$. Thus, offsets due to contact potential variations or similar effects are extremely important if it is desired to reach kinetic energies approaching those given by Eqs. (2), (10), and (13). Such problems can be alleviated by applying external voltages to the electrodes to compensate for any of the above type of asymmetries. This is easily accomplished along the $z$ axis by applying different static voltages to the two endcaps. In the $x$ and $y$ directions, separate compensating electrodes could be added or, for example, the ring could be split into quadrants. ${ }^{34}$ Different static voltages could then be applied to the quadrants in order to compensate for any asymmetric contact potential difference on the ring.

Next, we consider the effects of rf voltages which may be asymmetrically applied to the electrodes. This situation might occur, for example, because of different impedances from either endcap to ground. If the amplitude of the rf voltage on the two endcaps is different, this amounts to shifting the origin of the coordinate system for the rf voltage. In this new coordinate system, a voltage $U_{0}$ applied symmetrically to the endcap electrodes or to the ring electrode causes a static force which displaces the ion from the origin for the rf fields and yields a resultant micromotion which can be deduced from the previous arguments. A new problem to consider, however, is that the phase of the rf voltages on the electrodes might be different. To estimate the effects of this, assume that the voltage $V_{0} \cos (\Omega t+\theta / 2)$ is applied to one endcap and the voltage $V_{0} \cos (\Omega t-\theta / 2)$ is applied to the other endcap. The ring electrode is assumed to be grounded. If $\theta<<1$, we can consider the ion motion to be the sum of a solution for the condition when $V_{0} \cos (\Omega t)$ is applied to both endcaps and a perturbation to this solution for the additional voltages $\left(V_{0} \theta / 2\right) \sin (\Omega t)$ and $-\left(V_{0} \theta / 2\right) \sin (\Omega t)$ applied to the two endcaps, respectively. Classically, we can easily solve for the kinetic energy $E_{K \theta}$ due to the perturbed motion (averaged over $2 \pi / \Omega$ ). We have

$$
E_{K \theta}=\frac{m}{2}\left[\frac{\omega_{z}\left(r_{0}^{2}+2 z_{0}^{2}\right) \alpha \theta}{8 z_{0}}\right]^{2} \text {. }
$$

For our example of the ${ }^{198} \mathrm{Hg}^{+}$ion, ${ }^{7}$ we obtain $E_{K \theta} \simeq 2.4 \times 10^{8} \theta^{2} \hbar \omega_{z}$. Thus, very precise control of the phase of the $\mathrm{rf}$ voltage is required to reach micromotion kinetic energies near the zero-point energy of the secular motion. This can be accomplished by injecting a portion of the drive signal (with the proper phase) onto one endcap.

Because of the relatively strong effect of asymmetrically applied potentials, it is natural to ask whether imperfections in the electrode shapes can lead to residual rf motion 
even when the ion reaches the position given by $\langle\mathbf{F}\rangle=0$. To estimate the effects of electrode distortion, we must modify Eq. (19). About a suitable origin we can write

$$
\begin{aligned}
\phi(r, z)=\left[U_{0}+V_{0} \cos (\Omega t)\right]\left(\frac{r^{2}-2 z^{2}}{r_{0}^{2}+2 z_{0}^{2}}+A_{2}\left(x^{2}-y^{2}\right)\right. \\
+A_{3} x^{3}+B_{3} x^{2} y+C_{3} x^{2} z \\
\\
\left.+D_{3} x y^{2}+\cdots+D_{4} x^{4}+\cdots\right)
\end{aligned}
$$

where Laplace's equation is satisfied for each order. To estimate the effects of the higher-order terms, we examine the term $U_{0} C_{3} x^{2} z=U_{0} C_{3}^{\prime} x^{2} z / z_{0}^{3}$ in Eq. (24). Unless the electrodes are severely distorted, we have $C_{3}^{\prime}<1$. Near the origin, this term gives rise to a $z$ component of electric field $E_{z}^{\prime}=-\left(U_{0} / z_{0}\right) C_{3}^{\prime}\left\langle x^{2}\right\rangle / z_{0}^{2}$. If we can cool to near the zero-point energy $\left(\left\langle n_{x}\right\rangle<1\right)$, then $\left\langle x^{2}\right\rangle \simeq x_{0}^{2}$. For the conditions of a $\mathrm{Hg}^{+}$ion in a miniature rf trap, $\omega_{x} / 2 \pi=1.5 \mathrm{MHz}, z_{0}=320 \mu \mathrm{m}, x_{0} \simeq 4.1 \times 10^{-3} \mu \mathrm{m}$, $U_{0} \simeq 10 \mathrm{~V}$ (conditions for an approximately spherical trap). For $C_{3}^{\prime} \simeq 0.1$, the resulting field $E_{z}^{\prime}$ would be equivalent to applying a voltage $\Delta V \simeq 4 \times 10^{-10} \mathrm{~V}$ to one endcap. From Eq. (22), $E_{K \mu}(z) \simeq 1 \times 10^{-13} \hbar \omega_{z}$ for this value of $\Delta V$ which is negligible. Therefore, this effect by itself can be small. However, electrode distortion may give rise to unbalanced $\mathrm{rf}$ phases on the endcaps. Other terms in Eq. (24) appear to be of this order or smaller.

\section{SIDEBAND COOLING FOR A SINGLE ION IN A PENNING TRAP}

Sideband cooling for a single ion in a Penning trap requires special attention because of the peculiar properties of the magnetron motion. ${ }^{27}$ Cooling for the cyclotron and axial degrees of freedom is essentially the same as for the secular motion in the rf trap. However, for the magnetron motion, we will define cooling to be the reduction of the kinetic energy in this degree of freedom. This implies that the total energy of this degree of freedom increases, since most of the energy in the magnetron motion degree of freedom is due to electrical potential energy from the radially outward trap force. For positive charges, this potential energy is given by Eq. (19) with $V_{0}=0$ and $U_{0}<0$. In the axial direction, the ion is bound in a harmonic well, and in the radial direction the ion sits on a potential hill whose maximum is at the center of the trap where we choose $\phi=0$. First, consider the Doppler cooling limit $\left(\gamma \gg \omega_{m}, \omega_{c}^{\prime}, \omega_{z}\right.$ where $\omega_{m}, \omega_{c}^{\prime}$, and $\omega_{z}$ are the magnetron, cyclotron, and axial oscillation frequencies in the Penning $\left.\operatorname{trap}^{25,27}\right)$. To achieve cooling, we require that the ion preferentially absorb photons when it moves toward the laser due to cyclotron motion and simultaneously preferentially absorb photons when it moves away from the laser due to the magnetron motion. Since the cyclotron and magnetron motions are both in the $x-y$ plane, the only way this can be done is to use a spatially inhomogeneous laser beam. This case has been treated previously. ${ }^{27}$

In the sideband cooling limit $\left(\gamma<<\omega_{m}, \omega_{c}^{\prime}, \omega_{z}\right)$, we are able to use spatially homogeneous, plane-wave laser beams for the following reason. In Fig. 4, we show the absorption spectrum in the $x-y$ direction averaged over many scattering events for an ion that has been cooled within the Lamb-Dicke limit $\left\langle k^{2} x^{2}\right\rangle=k^{2} r_{0}^{2}\left(n_{c}+n_{m}\right.$ $+1) / 2<1$, where ${ }^{27} r_{0}=\left[2 \hbar / m\left(\omega_{c}^{\prime}-\omega_{m}\right)\right]^{1 / 2}$. In this limit, only the first-order sidebands are of appreciable size. The schematic representation of Fig. 4 may not be to scale, but the expressions for the relative intensities above the indicated sidebands should be valid. For this figure, we have assumed $\omega_{c}^{\prime}=5 \omega_{m} / 2$ in order to simplify the drawing. This diagram and the remarks above show that simultaneous sideband cooling of the magnetron and cyclotron degrees of freedom can be achieved by having two plane-wave laser beams directed normal to the $z$ axis. One laser beam can be tuned to $\omega_{0}-\omega_{c}^{\prime}$ and the other beam tuned to $\omega_{0}+\omega_{m}$. In principle, it should be possible to achieve cooling by having one laser tuned to the sideband at frequency $\omega_{0}-\omega_{c}^{\prime}+\omega_{m}$, but, in the Lamb-Dicke limit, the absorption intensity of this sideband is considerably reduced. Below, we examine the limits to sideband cooling for the Penning trap. However, we first note that sideband cooling will work only at relatively low temperatures in the Penning trap where particular sidebands are well resolved as shown in Fig. 4. At much higher temper-

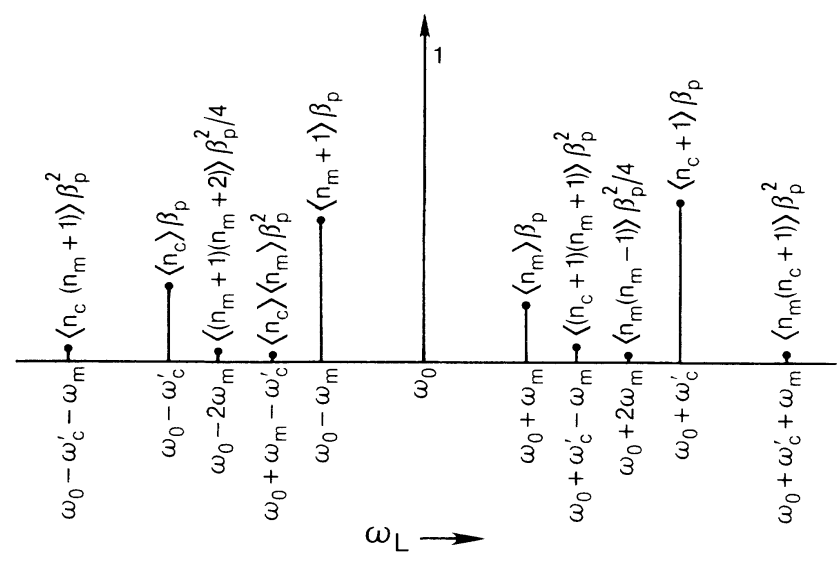

FIG. 4. Amplitudes of the lowest-order sidebands in the absorption spectrum for a single ion confined to the Lamb-Dicke region in a Penning trap. $\left(\omega_{c}^{\prime}=5 \omega_{m} / 2\right.$ is assumed. $)$ The radiation source (laser) is assumed to be directed normal to the $z$ axis. The carrier amplitude (at frequency $\omega_{0}$ ) is normalized to 1 . $k=\omega_{0} / c, r_{0}=\left[2 \hbar / m\left(\omega_{c}^{\prime}-\omega_{m}\right)\right]^{1 / 2}, \beta_{p}=\left(k r_{0}\right)^{2} / 4$ and $n_{c}$ and $n_{m}$ are the cyclotron and magnetron quantum numbers, respectively. Simultaneous cooling of the magneton and cyclotron degrees of freedom will occur for two laser beams tuned to $\omega_{0}+\omega_{m}$ and $\omega_{0}-\omega_{c}^{\prime}$, respectively. 
atures, the magnetron and cyclotron sideband distributions become nearly continuous, and it is impossible to simultaneously cool the kinetic energies for both degrees of freedom using plane waves. This implies the necessity of some form of precooling as discussed in Sec. IV.

The basic formalism for cooling of ions in Penning traps has already been established. ${ }^{27}$ In the quantum treatment of the ion motion, the total energies of the axial, cyclotron, and magnetron degrees of freedom can be written as $E_{z}=\left(n_{z}+\frac{1}{2}\right) \hbar \omega_{z}, \quad E_{c}=\left(n_{c}+\frac{1}{2}\right) \hbar \omega_{c}^{\prime}$, and $E_{m}=-\left(n_{m}+\frac{1}{2}\right) \hbar \omega_{m}$, respectively. The mean amplitudes of the motion we desire to reduce are given by $\left\langle z^{2}\right\rangle$ $=z_{0}^{2}\left\langle 2 n_{z}+1\right\rangle,\left\langle r_{c}^{2}\right\rangle=r_{0}^{2}\left\langle n_{c}+\frac{1}{2}\right\rangle$, and $\left\langle r_{m}^{2}\right\rangle=r_{0}^{2}\left\langle n_{m}\right.$ $\left.+\frac{1}{2}\right\rangle$, where $z_{0}=\left(\hbar / 2 m \omega_{z}\right)^{1 / 2}$ as in Sec. II. The axial cooling is identical to that described for the secular motion in the rf trap in Sec. II. Now, however, we are entirely free of the energy in the micromotion since the fields are static in the Penning trap. From Ref. 27 we can write a general expression for the average rate of change of the axial, cyclotron, and magnetron motion quantum numbers for the $\xi$ th laser beam (total intensity $I_{\xi}$ ) as

$\frac{d\left\langle n_{i}\right\rangle}{d t}=\sum_{\left\{n^{l}\right\},\left\{n^{f}\right\}} P\left(\left\{n^{l}\right\}\right) \Gamma\left(\left\{n^{l}\right\} \rightarrow\left\{n^{f}\right\}\right)\left(n_{i}^{f}-n_{i}^{l}\right)$.

In Eq. (25), $\left\{n^{l}\right\}=\left\{n_{z}^{l}, n_{c}^{l}, n_{m}^{l}\right\}$ is the set of initial (l=lower) quantum numbers, $\left\{n^{f}\right\}$ is the set of final ( $f=$ final) quantum numbers, and $i=z, c$, or $m . P$ is the initial probability distribution for quantum numbers $\left\{n^{l}\right\}$ $\left[P\left\{n^{l}\right\}\right.$ takes the role of $P\left(n_{l i}\right)$ from Sec. II $] . \Gamma$ is the transition rate given by

$$
\Gamma\left(\left\{n^{l}\right\} \rightarrow\left\{n^{f}\right\}\right)=\left(I_{\xi} / \hbar \omega_{0}\right) \sigma^{*}\left(\left\{n^{l}\right\} \rightarrow\left\{n^{f}\right\}\right) .
$$

In Eq. (26), $\sigma^{*}$ is the cross section given by

$$
\sigma^{*}\left(\left\{n^{l}\right\} \rightarrow\left\{n^{f}\right\}\right)=\sigma_{0}(\gamma / 2)^{2} \int\left|\sum_{\left\{n^{j}\right\}} \frac{\left\langle\left\{n^{f}\right\}\left|e^{-i \mathbf{k}_{s} \cdot \mathbf{r}}\right|\left\{n^{j}\right\}\right\rangle\left\langle\left\{n^{j}\right\}\left|e^{i \mathbf{k} \cdot \mathbf{r}}\right|\left\{n^{l}\right\}\right\rangle}{\omega_{0}-\omega-i \gamma / 2+\omega\left(\left\{n^{j}\right\}\right)-\omega\left(\left\{n^{l}\right\}\right)}\right|^{2} P_{s}\left(\hat{\mathbf{k}}_{s}\right) d \Omega
$$

In Eq. (27), $\mathbf{k}$ and $\mathbf{k}_{s}$ are the wave vectors of the incident and scattered light. $P_{s}\left(\hat{\mathbf{k}}_{s}\right) d \Omega$ is the probability that a photon is emitted into a solid angle $d \Omega$ in the $\widehat{\mathbf{k}}_{s}$ direction $\left(\hat{\mathbf{k}}_{s}=\mathbf{k}_{s} /\left|\mathbf{k}_{s}\right|\right)$, and $\hbar\left[\omega\left(\left\{n^{j}\right\}\right)-\omega\left(\left\{n^{l}\right\}\right)\right]$ is the difference in motional energy between the initial $(l)$ and intermediate $(j)$ states. We have

$$
f_{s i}=\int P_{s}\left(\hat{\mathbf{k}}_{s}\right)\left(\hat{\mathbf{k}}_{s i}\right)^{2} d \Omega
$$

where $\widehat{\mathbf{k}}_{s i}$ is the component of $\widehat{\mathbf{k}}_{s}$ in the $i$ th direction. In Eq. (27), we have assumed $\Delta \omega_{L} \ll<$. Equations (25) $-(27)$ reduce to Eq. (5) for the case of harmonic oscillator cooling or cooling the secular motion in an rf trap.

If we use Eqs. (25)-(27) and the operator relations ${ }^{11,27}$ for $\left[N_{i}, e^{i \mathbf{k} \cdot \mathrm{r}}\right]$, where $N_{i}=a_{i}^{\dagger} a_{i}$ is the operator whose eigenvalue is $n_{i}$, we obtain the following relationship for the cooling due to the $\xi$ th laser beam:

$$
\frac{d\left\langle n_{i}\right\rangle}{d t}=\dot{N}_{\xi} \int I_{\xi}^{\prime} d \omega \sum_{\left\{n^{l}\right\},\left\{n^{j}\right\}} P\left(\left\{n^{l}\right\}\right) \frac{\left\{\beta_{i} f_{s i}+\left[n_{i}(j)-n_{i}(l)\right]\right\}\left|\left\langle\left\{n^{j}\right\}\left|e^{i \mathbf{k}_{\xi^{\cdot}} \mathbf{r}}\right|\left\{n^{l}\right\}\right\rangle\right|^{2}}{1+\left[\frac{2}{\gamma}\left[\omega_{0}-\omega+\omega\left(\left\{n^{j}\right\}\right)-\omega\left(\left\{n^{l}\right\}\right)\right]\right]^{2}}
$$

In this expression, $\dot{N}_{\xi}=I_{\xi} \sigma_{0 \xi} / \hbar \omega_{0}$ and we have now allowed the laser spectral distribution to have some width as in Sec. II, such that $\int I_{\xi}^{\prime}(\omega) d \omega=1$. Also,

$$
\begin{aligned}
& \beta_{i}=\left\{\begin{array}{l}
\left(k r_{0}\right)^{2} / 4 \quad(i=c \text { or } m) \\
\left(k z_{0}\right)^{2} \quad(i=z),
\end{array}\right. \\
& f_{s i}=\left\{\begin{array}{l}
f_{s x}+f_{s y} \quad(i=c \text { or } m) \\
f_{s z}(i=z) .
\end{array}\right.
\end{aligned}
$$

Equation (28) looks very similar to Eq. (5) except that the recoil energy $\left(\beta_{i} f_{s i}\right.$ term) divides between the cyclotron and magnetron degrees of freedom in such a way that the average change of $\left\langle n_{m}\right\rangle$ or $\left\langle n_{c}\right\rangle$ is the same upon photon reemission (i.e., the incremental changes in $r_{c}$ and $r_{m}$ are the same).

In the Lamb-Dicke limit, Eq. (28) simplifies considerably because $e^{i \mathbf{k}_{\xi} \cdot \mathbf{r}} \simeq 1+\mathbf{k}_{\xi} \cdot \mathbf{r}$ and only matrix elements for transitions where $\Delta n_{i}=0, \pm 1$ need be considered. This is the same situation as for the harmonic oscillator case of Sec. II. However, for the Penning-trap case, both the cyclotron and magnetron cooling beams are assumed to be incident in the $x-y$ plane. Therefore, both beams cause transitions where $\Delta n_{m}=0, \pm 1$ and $\Delta n_{c}=0, \pm 1$, and some extra terms are encountered. For example, the contribution of $d\left\langle n_{i}\right\rangle / d t$ due to the cyclotron cooling beam (tuned to $\omega_{0}-\omega_{c}^{\prime}$ and assumed to be directed along the $y$ axis) is given by 


$$
\begin{aligned}
\frac{d\left\langle n_{i}\right\rangle}{d t} \simeq \dot{N}_{c} \sum_{\left\{n^{l}\right\}} P\left(\left\{n^{l}\right\}\right) & \left(\beta_{i} f_{s i}-\delta_{i c}\right)\left|\left\langle n_{c}-1\left|e^{i k y}\right| n_{c}\right\rangle\right|^{2} F_{c}+\beta_{i} f_{s i} \frac{\left|\left\langle n_{c}\left|e^{i k y}\right| n_{c}\right\rangle\right|^{2}}{\left(2 \omega_{c}^{\prime} / \gamma\right)^{2}} \\
& +\left(\beta_{i} f_{s i}+\delta_{i c}\right) \frac{\left|\left\langle n_{c}+1\left|e^{i k y}\right| n_{c}\right\rangle\right|^{2}}{\left(4 \omega_{c}^{\prime} / \gamma\right)^{2}}+\left(\beta_{i} f_{s i}-\delta_{i m}\right) \frac{\left|\left\langle n_{m}-1\left|e^{i k y}\right| n_{m}\right\rangle\right|^{2}}{\left[2\left(\omega_{c}^{\prime}+\omega_{m}\right) / \gamma\right]^{2}} \\
& \left.+\left(\beta_{i} f_{s i}+\delta_{i m}\right) \frac{\left|\left\langle n_{m}+1\left|e^{i k y}\right| n_{m}\right\rangle\right|^{2}}{\left[2\left(\omega_{c}^{\prime}-\omega_{m}\right) / \gamma\right]^{2}}\right] .
\end{aligned}
$$

As in Sec. II, $F_{c}=\int I_{c}^{\prime}(\omega)\left\{1+\left[2\left(\omega_{0}-\omega_{c}^{\prime}-\omega\right) / \gamma\right]^{2}\right\}^{-1} d \omega$, and we have assumed $\Delta \omega_{L} \ll<\omega_{m}, \omega_{z}, \omega_{c}^{\prime}$ for simplicity.

We can find the other two equations which are analogous to Eq. (30) for the magnetron cooling beam and axial cooling beam. By summing these equations, we arrive at expressions for the total rates of change of average quantum numbers for the three degrees of freedom $d\left\langle n_{i}\right\rangle / d t$ $(i=c, m$, or $z$ ). These expressions take the form

$$
\begin{array}{r}
d\left\langle n_{i}\right\rangle / d t=C_{i c}\left\langle n_{c}\right\rangle+C_{i m}\left\langle n_{m}\right\rangle+C_{i z}\left\langle n_{z}\right\rangle+d_{i} \\
(i=c, m, \text { or } z),
\end{array}
$$

where the coefficients $C_{i j}$ and $d_{i}$ come directly from Eq. (30) and the analogs for the cyclotron and magnetron beams. For steady state, $\left(d\left\langle n_{i}\right\rangle / d t=0\right)$ and we find that the $\left\langle n_{i}\right\rangle$ reach certain minimum values for given $\dot{N}_{\xi}$ and $F_{\xi}(\xi=c, m, z)$. These solutions can in general be quite complicated. To get some estimate for the minimum values for $\left\langle n_{i}\right\rangle$, we have solved these equations for the following simple conditions. We assume $f_{s x}=f_{s y}=f_{s z}$ $=\frac{1}{3}, \quad \omega_{m}<<\omega_{z}<<\omega_{c}^{\prime}$, and require a solution where $\left\langle n_{c}\right\rangle=\left\langle n_{m}\right\rangle=\left\langle n_{z}\right\rangle=\langle n\rangle$. For $F_{c}=F_{m}=F_{z}=F$, this implies the condition $\dot{N}_{c}=2 \dot{N}_{z}=8 \dot{N}_{m} / 11$ on the laser beam intensities and we obtain

$$
\langle n\rangle=(11 / 48 F)\left(\gamma / \omega_{m}\right)^{2}
$$

This has the same form as Eqs. (2), (10), and (13). For these solutions to hold, we have required $\beta \ll 1$ or $\omega_{R}<<\omega_{m}$ and $\omega_{m}<<\omega_{z}<<\omega_{c}^{\prime}<\omega_{c}$. These conditions may only be approximated in an experiment. To see this, we note that we can write $\omega_{R} / \omega_{c}=1.3 \quad(\lambda / 100$ $\mathrm{nm})^{-2} B^{-1} Z^{-1}$, where $B$ is in tesla, $Z$ is the ion charge (in units of the proton charge), $\omega_{c}$ is the unshifted cyclotron frequency, $\omega_{c}=q B / m c$, and $\lambda$ is the wavelength of the cooling laser radiation. To satisfy the above conditions, we require a large magnetic field and a relatively long wavelength cooling transition. A possible case might be $B^{+}\left[\lambda\left({ }^{1} S_{0} \rightarrow{ }^{3} P_{1}\right)=268 \mathrm{~nm}\right]$ at $15 \mathrm{~T}$. Here, $\omega_{R} / \omega_{c}$ $=0.012$, and $\omega_{m}$ and $\omega_{z}$ can be adjusted so that Eq. (32) should be reasonably accurate.

In practice, it is usually easier to make $\omega_{x}, \omega_{y}$, and $\omega_{z}$ in the rf trap be much larger than $\omega_{m}$ can be made in the Penning trap. Therefore, the conditions for sideband cooling are more easily satisfied in the rf trap than in the Penning trap. The limit on how high we can make $\omega_{m}$ is a limit on how large a magnetic field we can achieve, since we must always have $\omega_{m}<\omega_{z} / \sqrt{2}<\omega_{c}^{\prime}<\omega_{c}=q B / m c$ (conditions for stable trapping). From a comparison of
Eqs. (10) or (13) and Eq. (32), we might conclude that much lower values of the kinetic energy (above the zeropoint energy) can be obtained with the rf trap than with the Penning trap. This may indeed be the case for the secular motion of the rf trap, but, as we have seen at the end of Sec. II, the kinetic energy in the micromotion of the rf trap may be much more difficult to suppress. For the Penning trap, there is not an analogous problem to the micromotion since the imposed fields are static. Therefore, it may, in practice, be possible to obtain smaller values of the kinetic energy in the Penning trap.

\section{TWO-STAGE LASER COOLING}

To reach the cooling limits provided by sideband cooling [Eqs. (10), (13), or (32)], some sort of two-stage cooling may be necessary. This has already been referred to in Sec. III for the Penning trap, where simultaneous sideband cooling of the magnetron and cyclotron motions can only be effective at low enough temperatures where the Lamb-Dicke limit is approached. Therefore, to approach the Lamb-Dicke limit, it may first be necessary to reach a kinetic energy provided by the Doppler cooling limit on a strongly allowed electric dipole transition. For the Penning trap, this also implies the use of a spatially inhomogeneous cooling beam. ${ }^{27}$

The same restriction does not apply for the $\mathrm{rf}$ trap. In principle, it should be possible to tune to the first lower sideband (for $\gamma \ll<\omega_{v}$ ) and wait a sufficient time to reach the steady state given by Eq. (10) or (13). Initial cooling could be enhanced by tuning to the lth lower sideband where $l \omega_{v}$ is approximately equal to one-half of the Doppler linewidth at the initial temperature. ${ }^{11}$ The maximum cooling rate is given by $d E / d t \simeq-l \hbar \omega_{v} \gamma / 2$. To get an idea of the initial cooling rate, we can estimate the time $\Delta t$ it takes to reduce the ion temperature from $T_{1}=300 \mathrm{~K}$ to $T_{1} / 2$. We have $\Delta t \simeq 3 k_{B} T_{1} /\left(\hbar l \omega_{v} \gamma\right)$ $\simeq 3.8 \times 10^{4} / \gamma$, where we assume $l \omega_{v}=2 \pi(0.5) \mathrm{GHz}$. If the cooling transition is very weak (e.g., $\gamma \simeq 100 \mathrm{~s}^{-1}$ ), then $\Delta t \simeq 380 \mathrm{~s}$. Even under ideal conditions, this time is very long. Moreover, spurious heating mechanisms, such as collisional heating, may prevent any initial cooling at all. Thus, in the case of the rf trap as well as the Penning trap, there may be some motivation for using a precooling stage [e.g., cooling to the Doppler limit of Eq. (1) where $\gamma$ is due to a strongly allowed electric dipole transition].

To get some idea of how such two-stage cooling might work, we examine the case of cooling in the rf (or harmonic) trap. Assume that the initial cooling is performed 
TABLE I. Examples of $k\left(\left\langle x^{2}\right\rangle\right)^{1 / 2},\left\langle n_{v}\right\rangle$, and $\epsilon_{D 2}$ for a few ions of recent experimental interest. We have assumed that the ions are confined in a harmonic well with oscillation frequency $\omega_{v} / 2 \pi$ and are cooled to the Doppler cooling limit [Eq. (1)]. $\gamma / 2 \pi$ and $\lambda$ are the radiative linewidth and wavelength corresponding to the cooling transition, $M$ is the ion mass in atomic mass units, $k=2 \pi / \lambda,\left\langle n_{v}\right\rangle$ is the mean occupation number of the ion in the harmonic well [from Eq. (33)], and $\beta=\left(k x_{0}\right)^{2}=R /\left(\hbar \omega_{v}\right) .3 \epsilon_{D 2}$ is the magnitude of the fractional second-order Doppler shift for the $x, y$, and $z$ directions combined [three times the value in Eq. (35)]. If the ions are confined in an $\mathrm{rf}$ trap, the magnitude of the second-order Doppler shift is valid only for the secular motion contribution.

\begin{tabular}{|c|c|c|c|c|c|c|c|c|}
\hline Ion & $\begin{array}{l}\gamma / 2 \pi \\
(\mathbf{M H z}) \\
\end{array}$ & $\begin{array}{c}\lambda \\
(\mathrm{nm})\end{array}$ & $M(u)$ & $\begin{array}{l}\omega_{v} / 2 \pi \\
(\mathrm{MHz}) \\
\end{array}$ & $k\left(\left\langle x^{2}\right\rangle\right)^{1 / 2}$ & $\left\langle n_{v}\right\rangle$ & $\beta$ & $3 \epsilon_{D 2}$ \\
\hline $\begin{array}{c}{ }^{24} \mathrm{Mg}^{+} \\
\left(3 s^{2} S_{1 / 2} \rightarrow 3 p^{2} P_{1 / 2}\right)\end{array}$ & 43 & 280 & 24 & 1 & 2.14 & 21 & 0.106 & $6 \times 10^{-18}$ \\
\hline $\begin{array}{c}{ }^{24} \mathrm{Mg}^{+} \\
\left(3 s^{2} S_{1 / 2} \rightarrow 3 p^{2} P_{1 / 2}\right)\end{array}$ & 43 & 280 & 24 & 5 & 0.43 & 3.8 & 0.021 & $6 \times 10^{-18}$ \\
\hline $\begin{array}{c}{ }^{198} \mathrm{Hg}^{+} \\
\left(6 s^{2} S_{1 / 2} \rightarrow 6 p{ }^{2} P_{1 / 2}\right)\end{array}$ & 70 & 194 & 198 & 1.5 & 0.91 & 22.8 & 0.018 & $1.2 \times 10^{-18}$ \\
\hline $\begin{array}{c}{ }^{198} \mathrm{Hg}^{+} \\
\left(6 s^{2} S_{1 / 2} \rightarrow 6 p^{2} P_{1 / 2}\right)\end{array}$ & 70 & 194 & 198 & 15 & 0.091 & 1.8 & 0.0018 & $1.2 \times 10^{-18}$ \\
\hline $\begin{array}{c}{ }^{138} \mathrm{Ba}^{+} \\
\left(6 s^{2} S_{1 / 2} \rightarrow 6 p^{2} P_{1 / 2}\right)\end{array}$ & 21 & 493 & 138 & 1.5 & 0.24 & 6.5 & 0.004 & $5.1 \times 10^{-19}$ \\
\hline $\begin{array}{c}{ }^{113} \operatorname{In}^{+} \\
\left(5 s^{2} S_{0} \rightarrow 5 s 5 p^{3} P_{1}\right) \\
\end{array}$ & 0.3 & 231 & 113 & 0.1 & 1.0 & 1 & 0.33 & $9.0 \times 10^{-21}$ \\
\hline
\end{tabular}

on a strong transition (transition 1) where $\gamma_{1} \gg \omega_{v}$. In the Doppler cooling limit, the mean oscillation quantum number can be derived from $\left(\left\langle n_{v}\right\rangle+\frac{1}{2}\right) \hbar \omega_{v}=\hbar \gamma_{1} / 2$ or

$$
\left\langle n_{v}\right\rangle=\left(\gamma_{1} / \omega_{v}-1\right) / 2 .
$$

In the Doppler cooling limit, we can also write

$$
k\left(\left\langle x^{2}\right\rangle\right)^{1 / 2}=2 \pi\left(\hbar \gamma_{1} / 2 m\right)^{1 / 2} /\left(\lambda_{1} \omega_{v}\right) .
$$

In Table I, we have calculated $\left\langle n_{v}\right\rangle$ and $k\left(\left\langle x^{2}\right\rangle\right)^{1 / 2}$ from Eqs. (33) and (34) for some of the favored ions in recent experiments. This table shows that the Lamb-Dicke limit $\left[k\left(\left\langle x^{2}\right\rangle\right)^{1 / 2}<<1\right]$ is closely approached in the Doppler cooling limit.

If the Doppler cooling limit is first reached, the next step might be to drive a much weaker transition in the same ion (linewidth $\gamma_{2}<\omega_{v}$ ) where the sideband cooling limit applies. As an example, consider the case of ${ }^{198} \mathrm{Hg}^{+}$ whose relevant electronic structure is shown in Fig. 5. Initial laser cooling can be achieved ${ }^{7}$ by cooling on the ${ }^{2} S_{1 / 2} \rightarrow{ }^{2} P_{1 / 2}$ transition at $\lambda=194 \mathrm{~nm}$. Sideband cooling could be achieved by driving the ${ }^{2} S_{1 / 2} \rightarrow{ }^{2} D_{3 / 2}$ quadrupole transition at $\lambda=198 \mathrm{~nm}$ or the ${ }^{2} S_{1 / 2} \rightarrow{ }^{2} D_{5 / 2}$ quadrupole transition at $\lambda=281 \mathrm{~nm}$. We could also drive the twophoton transition to accomplish this; in this case the effective $\mathbf{k}$ vector of the transition is $\mathbf{k}^{\prime}=\mathbf{k}_{1}+\mathbf{k}_{2}$, where $\mathbf{k}_{1}$ and $\mathbf{k}_{2}$ are the wave vectors of the two photons required for the transition, so that if $\mathbf{k}^{\prime} \| \hat{\mathbf{x}}$, then $\beta_{x}$ $=\left(\left|\mathbf{k}_{1}+\mathbf{k}_{2}\right| x_{0}\right)^{1 / 2}$.

Suppose we tune the second cooling laser to the first lower sideband at frequency $\left(\omega_{02}-\omega_{v}\right)$. Equation (8) shows that the ion scatter rate on this sideband is equal to $\dot{N} n_{v} \beta F / 3$, where we assume the geometry of Sec. II A (case 3). For the minimum time to reach the sideband cooling limit, we require $\dot{N} n_{v} \beta F / 3 \simeq \gamma_{2} / 2$ for $n_{v}=1$. If much higher power is used, the scattering in the wings of the carrier increases while the cooling rate saturates. This would give a higher limit than that of Eq. (10) or (13).
Since each scattering event removes an energy $\hbar \omega_{v}$ from the harmonic oscillator state, a minimum time $\sim 2 \gamma_{2}^{-1}\left\langle n_{v}\right\rangle$ is required to reach the sideband cooling limit. Here, $\left\langle n_{v}\right\rangle$ is the value obtained from precooling. For the conditions of the second ${ }^{198} \mathrm{Hg}^{+}$example in Table I, we estimate a minimum time of approximately $80 \mathrm{~ms}$ for the ${ }^{2} S_{1 / 2} \rightarrow{ }^{2} D_{3 / 2}$ transition and $400 \mathrm{~ms}$ for the ${ }^{2} S_{1 / 2} \rightarrow{ }^{2} D_{5 / 2}$ transition. If the goal is to perform veryhigh-resolution spectroscopy on the ${ }^{2} S_{1 / 2} \rightarrow{ }^{2} D_{5 / 2}$ "clock" transition, we would like to perform the sideband cooling using the ${ }^{2} S_{1 / 2} \rightarrow{ }^{2} D_{3 / 2}$ transition because of the shorter cooling time required. (We designate the ${ }^{2} S_{1 / 2} \rightarrow{ }^{2} D_{5 / 2}$ transition as the clock transition because its narrow linewidth suggests that it could be used as a frequency standard or clock.) This still is not an optimum

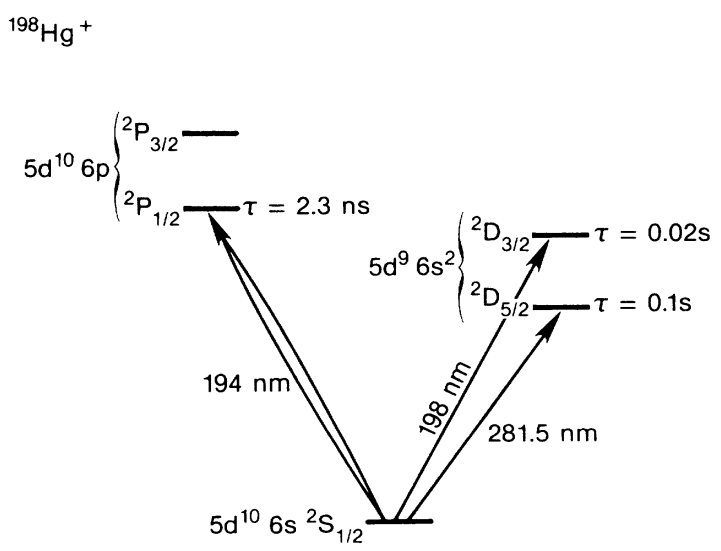

FIG. 5. Simplified energy-level diagram for ${ }^{198} \mathrm{Hg}^{+}$. The 281.5-nm quadrupole "clock" transition can be observed by monitoring the 194-nm fluorescence. If the ion has made a transition from the ${ }^{2} S_{1 / 2}$ to the ${ }^{2} D_{5 / 2}$ level the 194-nm fluorescence disappears. From Ref. 7. 
situation because we would like the cooling time to be much less than the interrogation time $\left(\sim \gamma_{2}^{-1}\right)$ for the clock transition in order to achieve maximum data rate and therefore best signal-to-noise ratio.

\section{MEASURING THE ZEROTH QUANTUM LEVEL}

We can verify that the sideband cooling limit given by Eqs. (10), (13), or (32) has been realized by measuring the spectrum of the sideband cooling transition. This corresponds to either the ${ }^{2} S_{1 / 2} \rightarrow{ }^{2} D_{3 / 2}$ or ${ }^{2} S_{1 / 2} \rightarrow{ }^{2} D_{5 / 2}$ transition in the $\mathrm{Hg}^{+}$example. To measure this spectrum (after cooling) we can use a simple double-resonance scheme. ${ }^{7,35,36}$ For the $\mathrm{Hg}^{+}$example, we can measure absorption on the ${ }^{2} S_{1 / 2} \rightarrow{ }^{2} D_{5 / 2}$ clock transition by monitoring the changes in fluorescence from the 194-nm cooling transition. ${ }^{7}$ In general, it will be necessary to drive the clock transition with the 194-nm laser switched off in order to avoid ac Stark shifts of the clock-transition spectrum. After the clock radiation is turned off, the fluorescence from the 194-nm cooling radiation will be absent if the clock transition has been made. If the ion remains in the ${ }^{2} S_{1 / 2}$ state after application of the $281-\mathrm{nm}$ radiation, then the fluorescence will immediately appear after admitting the 194-nm radiation. After each such detection cycle, the ion must be recooled by sideband cooling.

First, assume we measure the absorption spectrum along one of the principal axes $(x, y$, or $z)$ while staying below saturation intensity on the carrier. In the LambDicke limit, the strength of the lower sideband (assuming the carrier strength is equal to 1 ) is $\left\langle n_{v}\right\rangle \beta$ and that of the upper sideband is $\left(\left\langle n_{v}\right\rangle+1\right) \beta$. Here we assume that $n_{v}=n_{i}$ and $\beta=\beta_{i}$ for $i=x, y$, or $z$. If $\left\langle n_{v}\right\rangle \ll 1$, the lower sideband will be very small and the upper sideband will have amplitude $\beta$, as indicated qualitatively in Fig. 6(a). For the ${ }^{198} \mathrm{Hg}^{+}$examples of Table $\mathrm{I}, \beta$ is so small that the upper sideband may not be discernable above the

(a)

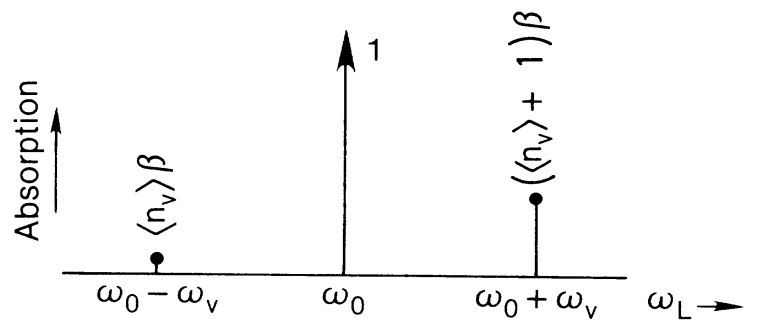

(b)

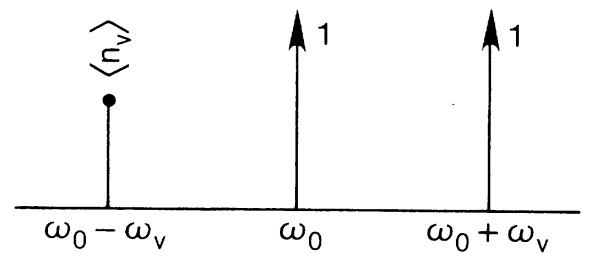

FIG. 6. Absorption spectrum of an ion in a harmonic potential for $\left\langle n_{v}\right\rangle<<1$ and $\beta=R / \hbar \omega_{v} \ll<$. In (a) relative amplitudes are given for the unsaturated spectrum. In (b) the radiation intensity is adjusted so that the upper sideband is just saturated. The amplitude of the weak lower sideband is a direct measure of $\left\langle n_{v}\right\rangle$. background. If, however, we increase the power so that we are just saturating the upper sideband, then the carrier and upper sideband have amplitude $\sim 1$ and the lower sideband has amplitude $\left\langle n_{v}\right\rangle$, as indicated qualitatively in Fig. 6(b). If we further increase the laser power by a factor of $K$, the carrier and upper sideband maintain an amplitude $\simeq 1$ and the lower sideband has amplitude $K\left\langle n_{v}\right\rangle$. By proper choice of $K$ [e.g., $\left.K \simeq 1 /\left(2\left\langle n_{v}\right\rangle\right)\right]$, we have a way to measure or put an upper limit on $\left\langle n_{v}\right\rangle$. Similar methods could be used to measure the amplitude of the micromotion of an ion in an rf trap.

\section{IMPLICATIONS FOR SPECTROSCOPY}

One of the original motivations for laser cooling was the reduction of Doppler shifts and broadening in veryhigh-resolution spectroscopy. ${ }^{37-39}$ Achieving the LambDicke limit essentially eliminates first-order Doppler broadening effects since the intensity in the sidebands is very small. This result is independent of temperature, but is easier to achieve at low temperatures. Historically, the uncertainty in the second-order Doppler frequency shift has been the primary limiting systematic effect in highresolution experiments on stored ions. This was true in the first high-resolution experiments on ${ }^{3} \mathrm{He}^{+}$ions ${ }^{40}$ (accuracy approximately equal to 1 part in $10^{9}$ ) and the most accurate experiments on ions to date, those on ${ }^{9} \mathrm{Be}^{+}$ ions $^{41}$ (accuracy approximately equal to 1 part in $10^{13}$ ). If the limits provided by sideband cooling can be realized, the second-order Doppler shift may no longer be the limiting systematic effect.

First, we note that even in the Doppler cooling limit for an allowed transition, the second-order Doppler shift would be extremely small. From Eq. (1), the limiting mean kinetic energy is given by $\hbar \gamma / 4$ for each degree of freedom. The Penning trap has one axial degree of freedom and two cyclotron degrees of freedom which contribute; therefore, the limiting kinetic energy is $3 \hbar \gamma / 4$. For the rf trap, the kinetic energy is at least twice this value due to the micromotion contribution (Sec. II B). The magnitude of the resulting minimum second-order Doppler shift per kinetic-energy degree of freedom $\epsilon_{D 2}$ is given by

$$
\epsilon_{D 2}=-\frac{\Delta v_{D 2}}{v_{0}}=\frac{\hbar \gamma}{4 m c^{2}} \simeq \frac{1.1 \times 10^{-18}[\gamma / 2 \pi(\mathrm{MHz})]}{M},
$$

where the right-hand side denotes the Doppler cooling limit. In Eq. (35), $\gamma / 2 \pi$ is expressed in $\mathrm{MHz}$ and $M$ is the ion mass in $u$ (atomic mass units). Values of $\epsilon_{D 2}$ for a few kinds of ions confined in a harmonic trap are given in Table I. $\epsilon_{D 2}$ gives the mean value of the second-order Doppler shift. The uncertainty in $\epsilon_{D 2}$ can be less depending on actual experimental conditions. In spite of the smallness of these numbers, it may be desirable to suppress the second-order Doppler shift further since other systematic effects may be controllable ${ }^{42}$ to levels below one part in $10^{18}$.

In the sideband cooling limit, since $\left\langle n_{v}\right\rangle<1$, the second-order Doppler shift is simply due to the kinetic energy in the zero-point oscillations $\hbar \omega_{v} / 4$ for each degree 
of freedom. Therefore,

$$
\begin{aligned}
\epsilon_{D 2}=-\frac{\Delta v_{D 2}}{v_{0}} & =\left(2\left\langle n_{v}\right\rangle+1\right) \frac{\hbar \omega_{v}}{4 m c^{2}} \\
& =\frac{1.1 \times 10^{-18}\left[\omega_{v} / 2 \pi(\mathrm{MHz})\right]}{M},
\end{aligned}
$$

where the right-hand side denotes the sideband cooling limit. $\epsilon_{D 2}$ is at least twice this value for the rf trap due to micromotion. Although these values of $\epsilon_{D 2}$ can be very small, the uncertainty in these values can be even lower. ${ }^{11}$ For example, the uncertainty in $\epsilon_{D 2}$ given by Eq. (36) might likely be due to the uncertainty of fluctuations in $\omega_{v}$. As an example, assume we perform sideband cooling of ${ }^{198} \mathrm{Hg}^{+}$ions in an $\mathrm{rf}$ trap where $\omega_{v} / 2 \pi=10 \mathrm{MHz}$. If the uncertainty in this oscillation frequency is $100 \mathrm{~Hz}$ and $\left\langle n_{v}\right\rangle \ll<10^{-5}$, the uncertainty in the second-order Doppler shift is $3.4 \times 10^{-24}$ ! It is not difficult to think about even much lower limits. If we go back to the expression for the ion temperature [Eq. (4)], we see that for fixed $\omega_{v}$, the temperature for the sideband cooling limit is not a strong function of $\gamma$ as it is for the Doppler cooling limit [due to the $\ln \left(\left\langle n_{v}\right\rangle^{-1}\right)$ factor]. In fact, for fixed $\gamma$, the Doppler cooling limit gives a lower temperature than the sideband cooling limit. However, as in the example above, the uncertainty in $\epsilon_{D 2}$ may be due to the uncertainty in $\omega_{v}$ and not directly a function of temperature. Thus, it would appear that temperature may not be a very useful parameter to describe the limits to high-resolution spectroscopy on bound atoms or ions.

With the potentially very low values of uncertainty in the second-order Doppler-shift correction, it continues to be an open question as to what will be the ultimate limit on accuracy in single-ion spectroscopy. The various effects of multipole electric and magnetic field interactions have to be considered; ${ }^{42}$ in principle, these effects could be measured and controlled to much less than 1 part in $10^{18}$. Certainly, practical issues now play an important role. At present, the required tunable narrow-band lasers do not exist, but recent experimental advances ${ }^{43}$ may provide a solution. If we assume that the spectral purity and amplitude stability of the lasers will not be a problem, we must still consider the fundamental limits to the signal-to-noise ratio. The high quantum multiplications achievable in double-resonance spectroscopy allow $100 \%$ detection efficiency. ${ }^{37}$ This has been demonstrated in recent experiments on single ions. ${ }^{7,44,45}$ In this case, the noise in the experiments is dominated by the shot noise in the clocktransition measurement. That is, the noise in the measurement process is governed by the fluctuations in whether or not the ion has undergone the clock transition after application of the clock radiation. If we assume $100 \%$ detection efficiency in the double-resonance experiment described in Sec. V (Fig. 6), the maximum signal-to-noise ratio for determining line center of the clock transition is given by measuring the transition probability at the halfpower points on both sides of the clock resonance and forcing the average frequency of the interrogating radiation to line center. If we make the assumption that the time-domain Ramsey method is used to interrogate the clock transition, then the frequency stability (two-sample
Allan variance ${ }^{46}$ ) is given as $^{37}$

$$
\begin{aligned}
\sigma_{y}(\tau) & \equiv\left[\left\langle\left(\left\langle\omega_{k}\right\rangle_{\tau}-\left\langle\omega_{k+1}\right\rangle_{\tau}\right)^{2}\right\rangle_{k} / 2 \omega_{0}^{2}\right]^{1 / 2} \\
& =\left(2 \omega_{0}^{2} T_{R} \tau\right)^{-1 / 2}
\end{aligned}
$$

In this expression, $\left\langle\omega_{k}\right\rangle_{\tau}$ is the $k$ th measurement of the frequency of the locked oscillator averaged over time $\tau$, \langle\rangle$_{k}$ denotes an average over many measurements, $\omega_{0}$ is the nominal frequency of the clock transition, and $T_{R}$ is the interrogation time between pulses in the Ramsey method. Thus $\sigma_{y}(\tau)$ is a measurement of the rms fluctuations of the frequency for measurement times $\tau$. Equation (37) assumes that optical state preparation (cooling and optical pumping) and double-resonance detection (from the 194-nm fluorescence signal in the example of Fig. 6) takes a time much less than $T_{R}$.

The accuracy of the measurement is limited to the value $\sigma_{y}(\tau)$ for a measurement time $\tau$. If, for example, $T_{R}=0.5 \mathrm{~s}$ [linewidth approximately equal to $\left(2 T_{R}\right)^{-1}=1$ $\mathrm{Hz}]$ and $\omega_{0} / 2 \pi=10^{15} \mathrm{~Hz}\left(\lambda_{2}=300 \mathrm{~nm}\right)$, then $\tau \simeq 2.5 \times 10^{4} \mathrm{~s}$ is required to make a measurement with an imprecision $\sigma_{y_{5}}(\tau)$ of 1 part in $10^{18}$. To reach a precision of about $10^{-25}$ would require a time equal to the age of the universe. Therefore, even though the intrinsic accuracy may be quite high, it may take a long averaging time to reach a comparable measurement precision. In other words, measurement precisions beyond 1 part in $10^{18}$ may require even higher $Q$ transitions (through smaller values of $\gamma$ ) and narrower linewidth lasers.

\section{CONCLUSIONS}

If the theoretical limits to laser cooling can be reached on a single trapped ion, the ion will nearly be held in the zeroth quantum level of its bound motion. This would be the closest possible realization of the ideal of a single isolated atom at rest. When cooling to near the zeroth quantum level is achieved, the temperatures achieved appear to lose their relevance; rather, we might be interested in the fluctuations of the zero-point energy due, for example, to fluctuations in the well depth of the trap.

In addition to the intellectual interest of achieving confinement to the zero-point energy state, the implications for accuracy in high-resolution spectroscopy are remarkable. For example, uncertainties in the second-order Doppler-shift correction might be $10^{-24}$ or lower. The possibility of these extremely low numbers clearly emphasizes the importance of many practical problems. For the rf trap, minimizing the kinetic energy in the micromotion may be a very difficult problem (Sec. II). For the Penning trap, no analog for the micromotion is apparent although it will be more difficult to obtain conditions where the sideband cooling limit applies (Sec. III). In addition, there is the fundamental problem of the limiting signal-to-noise ratio on a single ion; that is, we must ask, "Can we reach the required measurement precision in a reasonable length of time (Sec. VI)?" We must also consider the problems of laser spectral purity and the measurement of laser frequencies. Equally important, we 
must address the problems of controlling the various perturbations to the internal structure of the ions produced by external electric and magnetic fields. Nevertheless, in the end, it does not seem unreasonable to think that accuracies and measurement precisions at or beyond 1 part in $10^{18}$ will be achieved.

\section{ACKNOWLEDGMENTS}

We gratefully acknowledge the support of the U.S. Air Force Office of Scientific Research (AFOSR) and the Office of Naval Research (ONR). We also thank J. Cooper and L. Hollberg for helpful comments on the paper.
${ }^{1}$ The Mechanical Effects of Light, edited by P. Meystre and S. Stenholm [J. Opt. Soc. Am. B2, No. 11 (1985)].

${ }^{2}$ Atomic Physics 9, edited by R. S. Van Dyck, Jr., and E. N. Fortson (World Scientific, Singapore, 1985).

${ }^{3}$ Symposium Alfred Kastler, [Ann. Phys. (Paris) 10, No. 6 (1985)].

${ }^{4}$ S. Stenholm, Rev. Mod. Phys. 58, 699 (1986).

${ }^{5}$ W. Neuhauser, M. Hohenstatt, P. E. Toschek, and H. Dehmelt, Phys. Rev. A 22, 1137 (1980).

${ }^{6}$ W. Nagourney, G. Janik, and H. Dehmelt, Proc. Nat. Acad. Sci. (USA) 80, 643 (1983).

${ }^{7}$ J. C. Bergquist, R. G. Hulet, W. M. Itano, and D. J. Wineland, Phys. Rev. Lett. 57, 1699 (1986); J. C. Bergquist, W. M. Itano, and D. J. Wineland, Phys. Rev. A 36, 428 (1987).

${ }^{8}$ L. R. Brewer, J. D. Prestage, J. J. Bollinger, and D. J. Wineland, in Strongly Coupled Plasma Physics, NATO Advanced Study Institute, Series B: Physics, edited by J. Rogers and H. Dewitt (Plenum, New York, 1987), p. 53.

${ }^{9}$ S. Chu, L. Hollberg, J. E. Bjorkholm, A. Cable, and A. Ashkin, Phys. Rev. Lett. 55, 48 (1985).

${ }^{10}$ W. Neuhauser, M. Hohenstatt, P. Toschek, and H. Dehmelt, Phys. Rev. Lett. 41, 233 (1978).

${ }^{11}$ D. J. Wineland and W. M. Itano, Phys. Rev. A 20, 1521 (1979).

${ }^{12}$ R. S. Van Dyck, Jr., P. B. Schwinberg, and H. G. Dehmelt, in New Frontiers in High-Energy Physics, edited by B. M. Kursunoglu, A. Perlmutter, and L. F. Scott, (Plenum, New York, 1978), p. 159.

${ }^{13}$ S. P. Vyatchanin, Dokl. Akad. Nauk. SSSR 234, 1295 (1977); [Sov. Phys.-Dokl. 22, 321 (1977)]; L. S. Brown and G. Gabrielse, Rev. Mod. Phys. 58, 233 (1986).

${ }^{14}$ D. E. Pritchard, Phys. Rev. Lett. 51, 1336 (1983).

${ }^{15}$ S. Chu, J. E. Bjorkholm, A. Ashkin, J. P. Gordon, and L. Hollberg, Opt. Lett. 11, 73 (1986).

${ }^{16}$ S. Chu, J. E. Bjorkholm, A. Ashkin, L. Hollberg, and A. Cable, in Methods of Laser Spectroscopy, edited by Y. Prior, A. Ben-Reuven, and M. Rosenbluh (Plenum, New York, 1986), p. 41 .

${ }^{17}$ H. F. Hess, Bull Am. Phys. Soc. 30, 854 (1985).

${ }^{18}$ R. V. E. Lovelace, C. Mehanian, J. J. Tommila, and D. M Lee, Nature 318, 30 (1985).

${ }^{19}$ H. Dehmelt, G. Janik, and W. Nagourney, Bull. Am. Phys. Soc. 30, 111 (1985).

${ }^{20} \mathrm{~T}$. Sauter, W. Neuhauser, and P. E. Toschek, in Fundamentals of Laser Interactions, Vol. 299 of Lecture Notes in Physics, edited by F. Ehlotzky (Springer, Heidelberg, 1985).

${ }^{21}$ J. Javanainen, in Fundamentals of Laser Interactions, Vol. 299 of Lecture Notes in Physics, edited by F. Ehlotzky (Springer, Heidelberg, 1985) p. 249.

${ }^{22}$ V. G. Minogin and Yu. V. Rozhdestvensky, Appl. Phys. B 34, 161 (1984).

${ }^{23}$ M. Lindberg and J. Javanainen, J. Opt. Soc. Am. B 3, 1008 (1986).
${ }^{24}$ D. J. Wineland, in Precision Measurement and Fundamental Constants II, Natl. Bur. Stand. (U.S.) Special Publ. No. 617, edited by B. N. Taylor and W. D. Phillips, (U.S. GPO, Washington, D.C., 1984), p. 83.

${ }^{25}$ The basic concepts of ion trapping can be found in many references. See, for example, H. G. Dehmelt, Adv. At. Mol. Phys. 3, 53 (1967) and 5, 109 (1969); D. J. Wineland, W. M. Itano, and R. S. Van Dyck, Jr., ibid. 19, 135 (1983).

${ }^{26}$ M. Lindberg, J. Phys. B 17, 2129 (1984); J. Javanainen, M. Lindberg, and S. Stenholm, J. Opt. Soc. Am. B 1, 111 (1984).

${ }^{27}$ W. M. Itano and D. J. Wineland, Phys. Rev. A 25, 35 (1982).

${ }^{28}$ R. H. Dicke, Phys. Rev. 89, 472 (1953).

${ }^{29}$ R. J. Cook, D. G. Shankland, and A. L. Wells, Phys. Rev. A 31, 564 (1985).

${ }^{30}$ G. Janik, W. Nagourney, and H. Dehmelt, J. Opt. Soc. Am. B 2, 1251 (1985)

${ }^{31}$ D. J. Wineland and H. Dehmelt, J. Appl. Phys. 46, 919 (1975).

${ }^{32}$ G. Gabrielse, Phys. Rev. A 29, 462 (1984).

${ }^{33}$ E. C. Beaty, Phys. Rev. A 33, 3645 (1986).

${ }^{34}$ R. S. Van Dyck, Jr. and P. B. Schwinberg, Phys. Rev. Lett. 47, 395 (1981).

${ }^{35}$ H. G. Dehmelt, Bull. Am. Phys. Soc. 20, 60 (1975).

${ }^{36}$ D. J. Wineland and W. M. Itano, Phys. Lett. 82A, 75 (1981).

${ }^{37}$ D. J. Wineland, W. M. Itano, J. C. Bergquist, and F. L. Walls, in Proceedings of the Thirty-fith Annual Symposium on Frequency Control (1981), p. 602 (copies available from Electronics Industry Assn., 2001 "Eye" St., N.W. Washington, D.C. 20006).

${ }^{38}$ T. W. Hänsch and A. L. Schawlow, Opt. Commun. 13, 68 (1975).

${ }^{39}$ D. J. Wineland and H. Dehmelt, Bull. Am. Phys. Soc. 20, 637 (1975).

${ }^{40}$ E. N. Fortson, F. G. Major, and H. Dehmelt, Phys. Rev. Lett. 16, 221 (1966); F. G. Major and H. G. Dehmelt, Phys. Rev. 170, 91 (1968); H. A. Schuessler, E. N. Fortson, and H. G. Dehmelt, ibid. 187, 5 (1969).

${ }^{41}$ J. J. Bollinger, J. D. Prestage, W. M. Itano, and D. J. Wineland, Phys. Rev. Lett. 54, 1000 (1985).

${ }^{42}$ See, for example, H. G. Dehmelt, IEEE Trans. Instrum. Meas. IM-31, 83 (1982); D. J. Wineland, Science 226, 395 (1984), and references therein.

${ }^{43}$ J. Hall, C. Salomon, and D. Hils, in Proceedings of the Fourteenth International Conference on Quantum Electronics, Technical Digest (Optical Society of America, Washington, D.C., 1986), p. 80.

${ }^{44}$ W. Nagourney, J. Sandberg, and H. Dehmelt, Phys. Rev. Lett. 56, 2797 (1986).

${ }^{45}$ Th. Sauter, W. Neuhauser, R. Blatt, and P. E. Toschek, Phys. Rev. Lett. 57, 1696 (1986).

${ }^{46}$ J. A. Barnes, A. R. Chi, L. S. Cutler, D. J. Healey, D. B. Leeson, T. E. McGunigal, J. A. Mullen, Jr., W. L. Smith, R. L. Sydnor, R. F. C. Vessot, and G. M. R. Winkler, IEEE Trans. Instrum. Meas. IM-20, 105 (1971). 